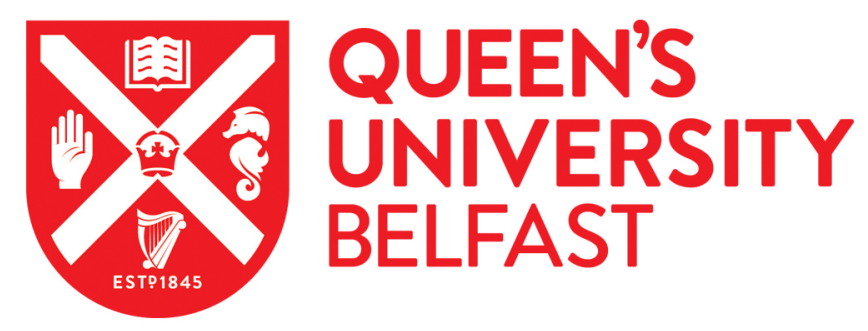

\title{
Arching in Concrete Slabs Strengthened with Near Surface Mounted Fibre Reinforced Polymers
}

\author{
Martin, A., Taylor, S., Robinson, D., \& Cleland, D. (2019). Arching in Concrete Slabs Strengthened with Near \\ Surface Mounted Fibre Reinforced Polymers. Engineering Structures, 184, 257-277. \\ https://doi.org/10.1016/j.engstruct.2019.01.076
}

\author{
Published in: \\ Engineering Structures
}

\section{Document Version:}

Peer reviewed version

Queen's University Belfast - Research Portal:

Link to publication record in Queen's University Belfast Research Portal

\section{Publisher rights}

Copyright 2019 Elsevier.

This manuscript is distributed under a Creative Commons Attribution-NonCommercial-NoDerivs License

(https://creativecommons.org/licenses/by-nc-nd/4.0/), which permits distribution and reproduction for non-commercial purposes, provided the author and source are cited.

\section{General rights}

Copyright for the publications made accessible via the Queen's University Belfast Research Portal is retained by the author(s) and / or other copyright owners and it is a condition of accessing these publications that users recognise and abide by the legal requirements associated with these rights.

Take down policy

The Research Portal is Queen's institutional repository that provides access to Queen's research output. Every effort has been made to ensure that content in the Research Portal does not infringe any person's rights, or applicable UK laws. If you discover content in the Research Portal that you believe breaches copyright or violates any law, please contact openaccess@qub.ac.uk. 


\title{
Arching in Concrete Slabs Strengthened with Near Surface Mounted Fibre Reinforced Polymers
}

\begin{abstract}
This paper outlines basalt fibre reinforced polymer (BFRP) and carbon fibre reinforced polymer (CFRP) strengthening of laterally restrained concrete floor slabs. In-plane restraint has previously been shown to enhance slab capacity due to the development of internal compressive membrane action (CMA), which is not generally included in codified strength assessments. By installing fibre reinforced polymers (FRPs) using the near surface mounted (NSM) technique, disturbance to the existing structure can be minimised. The span-to-depth ratios of test slabs were 20 and 15 and these were constructed with normal strength concrete $\left(\sim 40 \mathrm{~N} / \mathrm{mm}^{2}\right)$ with $0.15 \%$ steel reinforcement. $0.10 \%$ FRP (either BFRP or CFRP), was used to strengthen samples which were then compared with control samples. Investigations showed that FRP strengthening and CMA are generally separate, with limited overlap in terms of their contribution to capacity increase. Recommendations are then made for designers to better determine the capacity of FRP strengthened restrained slabs.
\end{abstract}

\section{Keywords}

Fibre reinforced polymer, carbon fibre reinforced polymer, FRP, BFRP, CFRP, strengthening, concrete, in-plane lateral restraint, near surface mounted, NSM, arching, compressive membrane action.

\section{Introduction}

It has been estimated that $87 \%$ of buildings which will be in existence in 2050 have already been built [1] and that $40 \%$ of global greenhouse gases are directly attributable to the built environment [2]. Therefore, 'adaptive reuse' has grown in popularity in recent years as a major means for the construction industry to be more sustainable [3] [4], with the life cycle considerations of repurposing buildings resulting in $20-41 \%$ savings in energy and resource consumption [5]. This ethos of repurposing structures has a wide range of applications ranging from strengthening existing structural elements by retrofitting, complete replacement of structural elements, the construction of new structural elements within an existing building (e.g. shear walls, 
steel bracing systems, etc.) and the application of modern insulating materials to enhance operational energy usage.

One potential application of adaptive reuse within structural engineering may be to increase the intensity of loading on floor slabs above that considered in their original design (e.g. changing from a domestic floor loading to light office floor loading, etc.). In the past, such a change of use may have resulted in demolition of the original structure and replacement with a new building incurring considerable financial outlay and pollution due to construction and demolition waste [6]. However, the use of advanced materials and innovative methods of analysis can provide engineers with an opportunity to deliver greater material efficiency and provide end users with a sustainable alternative to demolition and new construction. In recent years [7-11], the use of fibre reinforced polymers (FRPs) in retrofitting existing reinforced concrete structures has increased in popularity. This has typically been employed in strengthening highway bridges [12] due to the resistance of FRPs to corrosion from road de-icing salts. However, they can also be applied to multi-storey building frame elements [13] [14]. In most cases, strengthening has been carried out using conventional adhesive application (CAA) [15-17], near surface mounting (NSM) [18] [19] or by plate fastening [20]. FRPs have relatively low weight and good corrosion resistance and their application using the NSM technique involves minimal intrusion within the structure and minimises exposure to fire, which is seen as particularly advantageous in situations involving the structural retrofit of multi-storey buildings [21].

Of further benefit with regard to slab capacity increases is the inclusion of restraint and internal arching effects, which are not typically considered by designers but which may allow the quantification of additional capacity. Methods to quantify arching effects have been developed since the early part of the $20^{\text {th }}$ century [22-28] and a range of approaches are now available. This research makes particular use of the arching theory developed at Queen's University Belfast [29-36]. However, arching theories have not been incorporated within modern European or American building design codes, although some specialist highway design codes do allow their use in bridge deck design.

While the individual strength enhancing characteristics of FRP strengthening and arching have been well known for many years, a review of the literature has shown that no research into the simultaneous combination of the two methods has been carried out. Hence, this research outlines the investigations carried out to quantify the 
benefits of each approach acting concurrently and to provide a safe means for design engineers to apply them in practice.

\subsection{Background}

\subsubsection{NSM, BFRP and CFRP}

NSM strengthening of existing reinforced concrete structures can be traced back to strengthening bridge slabs with grouted steel reinforcement in 1949 [37] and whilst strengthening using steel bars continues to be of interest [38], the use of FRPs has gained interest more recently (e.g. [39]). Some bridges have also been built entirely or partially from FRP [40]. FRPs also offer faster construction, higher strengths, lower weights, and greater environmental durability compared with steel. However, the main perceived drawbacks are their higher initial cost and their lower elastic moduli compared to steel.

Basalt fibres are generated by melting basalt; which is one of the most common rocks found in the earth's crust; at $1300-1700{ }^{\circ} \mathrm{C}$ and spinning the molten liquid [41] into thin fibres. However, their mechanical properties are dependent, to an extent, on the origin of the raw material and the exact production processes employed. Carbon fibres were first produced in 1958 [42] during carbon arc experimentation under high temperatures and pressures [43] and since their original discovery industrial methods to produce them have been refined. FRP bars containing carbon or basalt fibres are then typically manufactured with either circular or rectangular cross sections using a pultrusion process to suspend the fibres within a polymer resin.

\subsubsection{Compressive membrane action}

If the edges of a concrete slab are restrained against lateral movement, internal arching develops as the slab deflects, as shown in Figure 1. This arching behaviour is known as compressive membrane action (CMA) and has been shown to enhance the flexural and shear capacity of reinforced concrete slabs.

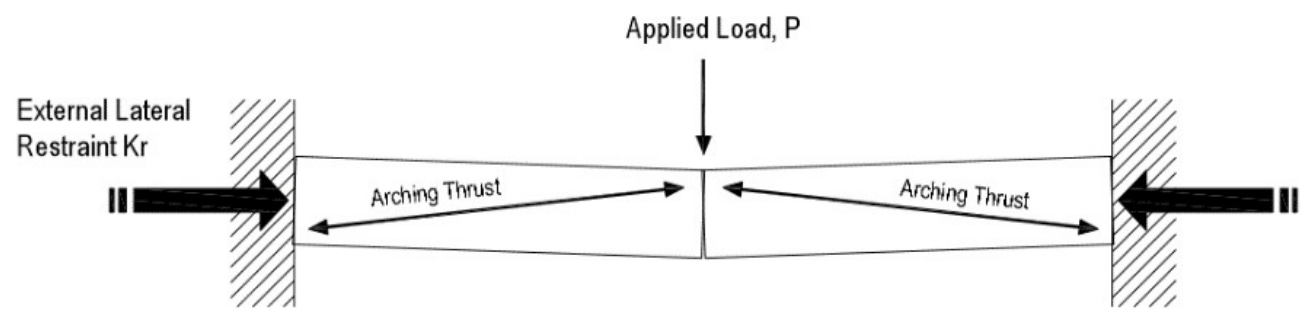

Figure 1: Arching Action in Laterally Restrained Slabs 
In the early part of the 20th century, the strength enhancing effects of arching action, above those predicted by flexural analysis, were first recognised [22]. However, it was not until the 1950s when full scale destructive tests were carried out [23] [24] that serious attempts to quantify arching were made. Since then, theories have been developed to explain arching, primarily by McDowell et al. [25] and Park [26-28]. More recently, researchers at Queen's University Belfast [29-36] and have built on these investigations.

\subsubsection{Queen's University of Belfast (QUB) Arching Theory}

The QUB arching theory [29] [30] equates a restrained three-pinned arch, with 'spring' restraints to a rigidly restrained three-pinned arch with a longer effective span, as shown in Figure 2.

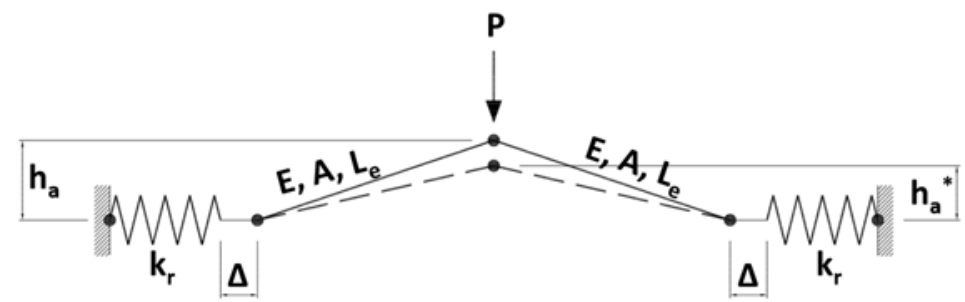

Figure 2(a): Elastically restrained three pinned arch [29]

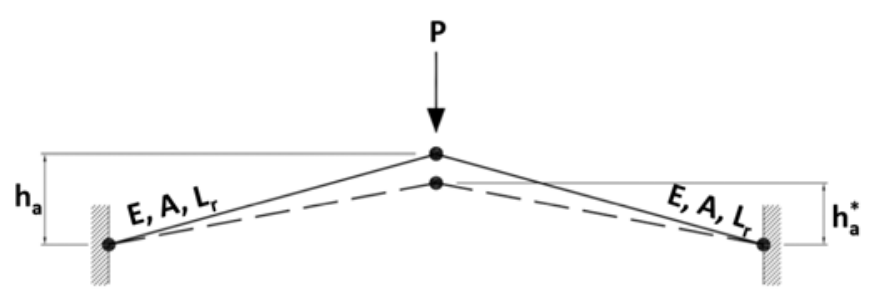

Figure 2(b): Equivalent rigidly restrained three pinned arch [29]

The theory for the prediction of ultimate capacity was based on the deformation theory of McDowell et al. [25] and the effects of arching and bending were considered separately, although in reality compression in concrete was due to the action of both arching and bending. This arching analysis was further developed [31] [32] for bridge deck slabs with high strength concrete $\left(>70 \mathrm{~N} / \mathrm{mm}^{2}\right)$.

Using Rankin's [29] relationship, a rigidly restrained three pinned arch was equated to that of an elastically restrained system, as illustrated in Figure 2 and defined in equation (1). However, as the main focus of this research was on the application of 
the arching theory to existing reinforced concrete slabs rather than a further development of the arching theory itself, a full explanation of the development of the arching theory is not included in this research but can be found in [36].

$$
L_{r}=L_{e}\left[\frac{E_{c} A}{k_{r} L_{e}}+1\right]^{1 / 3}
$$

where: $\quad L_{r}=$ Half span of equivalent rigidly restrained slab strip

$L_{e}=$ Half span of 'real' strip of slab with finite lateral restraint

$A=$ Area of concrete due to arching

$k_{r}=$ Stiffness of elastic spring restraint

Tests have previously shown good correlation between the QUB arching theory and experimental values [35] [36]. Also, in recent years, several international bridge design codes [44 - 46] have incorporated design guidance to include the beneficial effects of CMA in bridge deck slab design. The procedure for assessing the strength of laterally restrained slabs using the QUB arching theory is outlined below:

\section{Calculating the strength of an in-plane restrained slab}

The process of establishing the depth available for arching is iterative. Hence, the flow chart in Figure 3 illustrates the process involved in evaluating the strength of a laterally restrained slab.

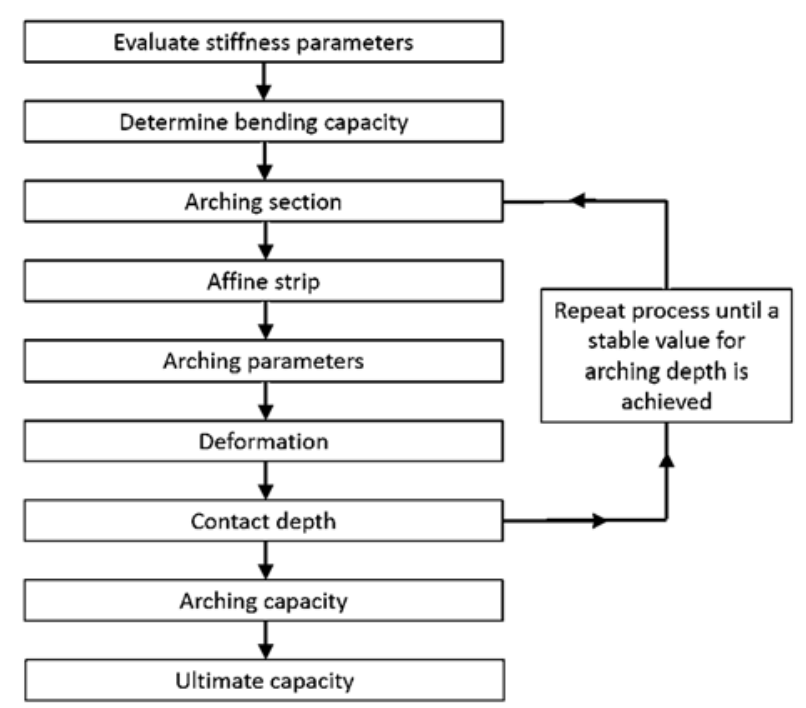

Figure 3: Flow chart illustrating iterative procedure to determine in-plane restrained slab capacity 


\section{Step 1}

\section{Stiffness parameters}

Evaluation of the restraint stiffness, $k_{r}$, in experimental slab specimens due to the presence of in-plane restraint beams was based upon an analysis of electrical resistance strain (ERS) gauge readings within restraint beam reinforcement bars and slab movements.

\section{Bending capacity}

Bending capacity of the rectangular cross section is based upon the original approach developed by Rankin [29] and Taylor [31], with the additional inclusion of FRP bars along with steel reinforcement, as shown in Figure 4.

Proportional depth of stress block factor: $\quad \beta=\mathbf{1}-\mathbf{0 . 0 0 3} f_{c k, c u b e}$ but $\leq \mathbf{0 . 9}$

Depth of neutral axis:

$$
x=\frac{A_{S} f_{y k}+A_{F R P} f_{F R P, k}}{0.67 f_{c k, c u b e} \beta b}
$$

Moment capacity due to bending:

$$
M_{b}=A_{S} f_{y k}\left[d-\frac{\beta x}{2}\right]+A_{F R P} f_{F R P, k}\left[d_{F R P}-\frac{\beta x}{2}\right]
$$

In all cases, the partial safety factors for steel and FRP materials are unity.

As loads are considered as midspan knife edge loads, the bending moment $\mathrm{M}_{\mathrm{b}}$ can be related to an equivalent knife edge load, $\mathrm{P}_{\mathrm{b}}$, using equation (5).

$$
P_{b}=\frac{4 M_{b}}{L}
$$
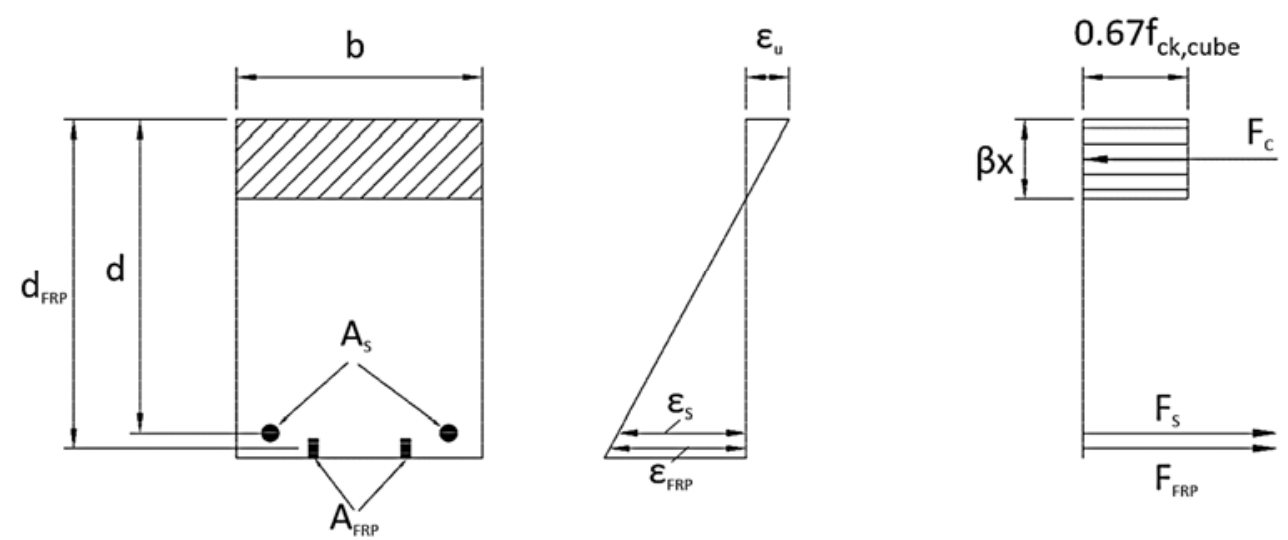

Figure 4: Rectangular section stress distribution for bending component within QUB Arching 


\section{Step 2}

\section{Arching section}

Depth available for arching, established by iteration: $d_{1}=\frac{h-2 \beta x}{2}$

\section{Affine strip}

Area of concrete due to arching: $\quad \boldsymbol{A}=\boldsymbol{\alpha b d _ { 1 }}$

$$
\alpha=1-\frac{u}{2}
$$

where: $\alpha=1$ for the first iteration, which is reflective of zero deflection $u=$ McDowell's [25] non-dimensional arching deflection parameter

Equivalent rigid half arch span: $\quad L_{r}=L_{e}\left[\frac{E_{C} A}{k_{r} L_{e}}+1\right]^{1 / 3}$

where: $L_{e}=$ Half of the actual slab span

$k_{r}=$ Axial restraint stiffness

\section{Arching parameters}

Ultimate compressive strain in concrete:

$$
\varepsilon_{u}=0.0043-\left[f_{c k, c u b e}-60\right] \times\left(2.5 \times 10^{-5}\right) \quad \text { but } \leq 0.0043
$$

Concrete plastic strain: $\quad \varepsilon_{c}=2 \varepsilon_{u}(1-\beta)$

McDowell's [25] non-dimensional geometry and material factor:

$$
R=\frac{\varepsilon_{c} L_{r}^{2}}{4 d_{1}^{2}}
$$

\section{Deformation}

$$
\begin{array}{lll}
\text { For } & 0<R \leq 0.26 & u=-0.15+0.36 \sqrt{0.18+5.6 R} \\
\text { For } & R>0.26 & u=0.31
\end{array}
$$

\section{Contact depth}

With a value of $u$ established, it is then possible to determine a refined value for the contact depth from equation (8), area of concrete due to arching from equation (7), 
equivalent rigid half arch span from equation (9), McDowell's non-dimensional geometry and material factor from equation (12) and back to a newly refined value for the contact depth from equation (8) before the iterative process repeats until equilibrium is established.

\section{$\underline{\text { Step } 3}$}

\section{Arching capacity}

For $\quad 0<R \leq 0.26, \quad$ Moment ratio $\quad M_{r}=4.3-16.1 \sqrt{\left(3.3 \times 10^{-4}\right)+0.1243 R}$

For $\quad R>0.26, \quad$ Moment ratio $\quad M_{r}=\frac{0.3615}{R}$

The equivalent rigid arching moment of resistance is expressed as:

$$
M_{a r}=0.168 b f_{c k, c u b e} d_{1}^{2} M_{r}\left(\frac{L_{e}}{L_{r}}\right)
$$

However, the elastic arching moment of resistance is expressed as:

$$
M_{a}=M_{a r}\left(\frac{L_{e}}{L_{r}}\right)
$$

As loads are considered as midspan knife edge loads, the bending moment due to arching, $M_{a}$, can be related to an equivalent knife edge load, $P_{a}$, using equation (19).

$$
P_{a}=\frac{4 M_{a}}{L}
$$

Ultimate capacity

$$
\boldsymbol{P}_{p}=\boldsymbol{P}_{\boldsymbol{a}}+\boldsymbol{P}_{b}
$$

\subsection{Objectives of the research}

The objective of the research was to investigate and quantify the benefits of using FRP strengthening in the presence of internal arching effects due to in-plane restraint which exist within many reinforced concrete framed buildings. However, these membrane effects are invariably ignored by practicing design engineers as a result of an unfamiliarity with their quantification. Therefore, this research also aims to provide a simplified means of estimating the level of restraint stiffness in both unstrengthened and FRP-strengthened slabs for application within the existing arching theory previously developed at Queen's University Belfast [29-36]. 


\section{Experimental Investigations}

To investigate the development of in-plane restraint in concrete slabs, it was necessary to develop an experimental programme which involved the production of a series of seventeen one third scale test slabs with varying levels of in-plane restraint, both with and without FRP strengthening. These were composed of concrete with a mix design based on previous studies by Zheng [47] at Queen's University Belfast. In the case of restrained slabs, in-plane restraint was determined from an analysis of slab restraining beam strains and their corresponding stresses using established tensile and compressive constitutive relationships.

Due to the variability of its material properties, concrete was tested in both tension and compression for each individual test slab. Tensile material tests were also carried out on representative test batches of all reinforcing steel, CFRP and BFRP strengthening bars used throughout the research. The following subsections outline these tests and their corresponding results.

\subsection{Material Properties}

\subsubsection{Concrete}

Normal strength concrete with a target strength of $40 \mathrm{~N} / \mathrm{mm}^{2}$ was used throughout the research, with the one third scale mix outlined in Table 1 [47]. The use of $6 \mathrm{~mm}$ aggregate avoided problems associated with size effects on shear behaviour and cracking in one-third scale test slabs.

Table 1: Concrete Mix Design [47]

\begin{tabular}{|c|c|c|c|c|c|c|}
\hline $\begin{array}{l}\text { Water } \\
\left(\mathrm{kg} / \mathrm{m}^{3}\right)\end{array}$ & $\begin{array}{l}\text { Cement } \\
\left(\mathrm{kg} / \mathrm{m}^{3}\right)\end{array}$ & $\begin{array}{c}6 \mathrm{~mm} \\
\text { Aggregate } \\
\left(\mathrm{kg} / \mathrm{m}^{3}\right)\end{array}$ & $\begin{array}{c}\text { Coarse } \\
\text { Grit } \\
\left(\mathrm{kg} / \mathrm{m}^{3}\right)\end{array}$ & $\begin{array}{l}\text { Zone } 2 \\
\text { Sand } \\
\left(\mathrm{kg} / \mathrm{m}^{3}\right)\end{array}$ & $w / b$ & $\begin{array}{c}\text { Target 28-Day Cube } \\
\text { Compressive } \\
\text { Strength (MPa) }\end{array}$ \\
\hline 250 & 400 & 525 & 875 & 350 & 0.625 & 40.00 \\
\hline
\end{tabular}

For each batch of concrete produced in the preparation of test slabs, slump tests were carried out in accordance with [48]. In addition, compressive cube tests and tensile splitting tests were carried out on hardened concrete in accordance with [49 - 51] using a calibrated testing machine [52]. 
Compressive strength tests were carried out on control batches of three $100 \mathrm{~mm}$ cubes at the time of slab testing and strengths, $f_{c k, c u b e}$, were established for each sample using equation (21) and which are outlined in Table 5.

$$
f_{c k, c u b e}=\frac{F}{100^{2}}
$$

where: $\quad f_{c k, c u b e}=$ Compressive cube strength of concrete $\left(\mathrm{N} / \mathrm{mm}^{2}\right)$

$$
F=\text { Maximum load at failure }(\mathrm{N})
$$

Tensile strengths, $f_{c t}$, were established by averaging values obtained from tensile splitting tests carried out on $200 \mathrm{~mm}$ long $\times 100 \mathrm{~mm}$ diameter cylinder samples in accordance with [51] and were established using equation (22) and which are outlined in Table 5.

$$
f_{c t}=\frac{2 F}{\pi L d}
$$

where: $\quad f_{c t}=$ Tensile splitting strength of concrete

$F=$ Maximum load $(\mathrm{N})$

$L=$ Length of cylinder $(\mathrm{mm})$

$d=$ Diameter of cylinder

\subsubsection{Steel reinforcement}

$6 \mathrm{~mm}$ diameter straight bars, $6 \mathrm{~mm}$ diameter bars from mesh and $32 \mathrm{~mm}$ diameter straight bars were used throughout the research. All bars were 'ribbed' [53] and $500 \mathrm{~mm}$ long representative samples were tested in tension at $0.2 \mathrm{kN} / \mathrm{s}$ [54] in batches

\begin{tabular}{|c|c|c|c|c|c|}
\hline Bar Diameter & $\begin{array}{c}\text { Upper } \\
\text { Yield, } R_{\mathrm{eH}} \\
\left(\mathrm{N} / \mathrm{mm}^{2}\right)\end{array}$ & $\begin{array}{l}\text { Lower Yield, } R_{\mathrm{el}} \\
\quad\left(\mathrm{N} / \mathrm{mm}^{2}\right)\end{array}$ & $\begin{array}{c}0.2 \% \text { proof } \\
\text { strength, } \mathrm{R}_{\mathrm{p} 0.2} \\
\left(\mathrm{~N} / \mathrm{mm}^{2}\right)\end{array}$ & $\begin{array}{c}\text { Yield } \\
\text { Strength, } f_{\mathrm{yk}} \\
\left(\mathrm{N} / \mathrm{mm}^{2}\right)\end{array}$ & $\begin{array}{c}\text { Elastic } \\
\text { modulus, } \mathrm{E}_{\mathrm{s}} \\
\left(\mathrm{N} / \mathrm{mm}^{2}\right)\end{array}$ \\
\hline $6 \mathrm{~mm}$ (Straight) & 499 & 483 & - & 499 & $205.5 \times 10^{3}$ \\
\hline $6 \mathrm{~mm}$ (Mesh) & - & - & 549 & 549 & $201.9 \times 10^{3}$ \\
\hline $32 \mathrm{~mm}$ (Straight) & - & - & - & 485 & $200 \times 10^{3}$ \\
\hline
\end{tabular}
of six within a universal testing machine. Average results are summarised in Table 2.

Table 2: Reinforcement properties 


\subsubsection{FRP bars}

For each FRP bar type, $500 \mathrm{~mm}$ long representative samples were tested in batches of six under tension at $0.2 \mathrm{kN} / \mathrm{s}$ within a universal testing machine, with attached electronic resistance strain (ERS) gauges [54]. Furthermore, an optical microscope with $\times 1000$ magnification was used to measure the cross sectional areas of both BFRP and CFRP bars from specially prepared thin samples, as shown in Figure 5. BFRP bars were composed of two central BFRP rods of approximately $2.5 \mathrm{~mm}$ diameter whilst CFRP bars were composed of three CFRP rods of approximately $2.1 \mathrm{~mm}$ diameter, as shown in Figure 6. In each case the individual rods were held together by helical thread and coated with an epoxy resin and sand coating. Averaged FRP properties are summarised in Table 3.

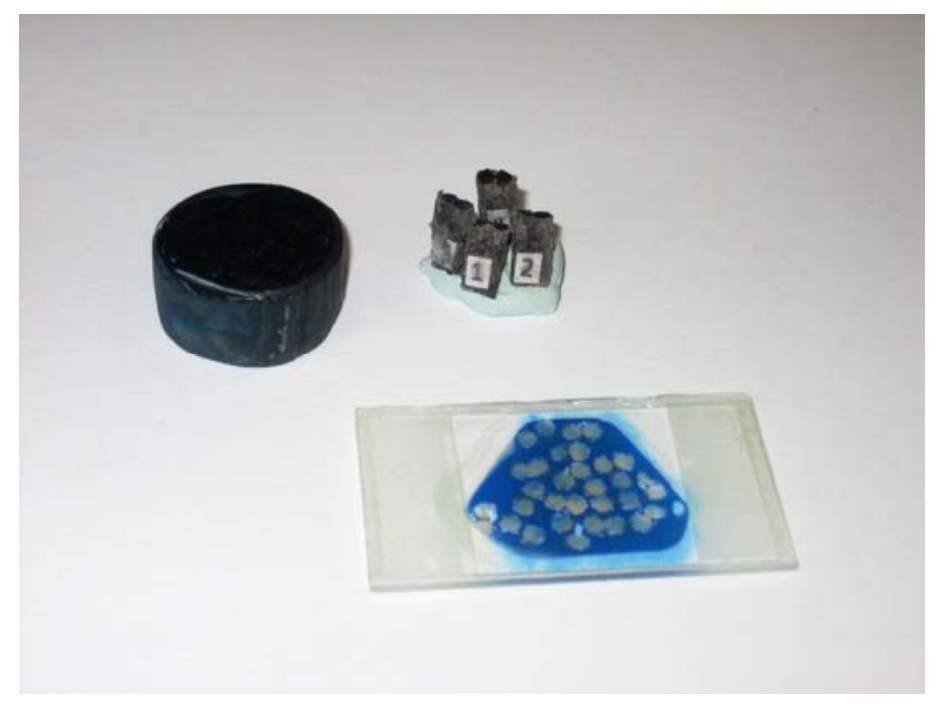

Figure 5: Example of FRP microscopy sample prepared from representative batch specimens

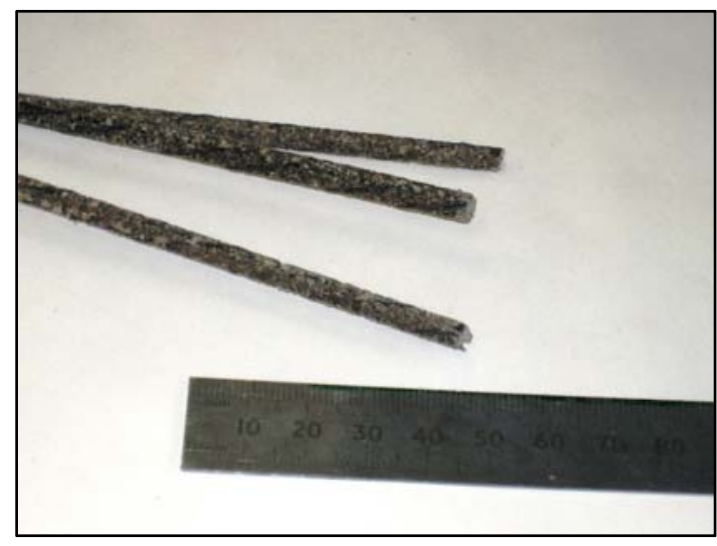

(a)

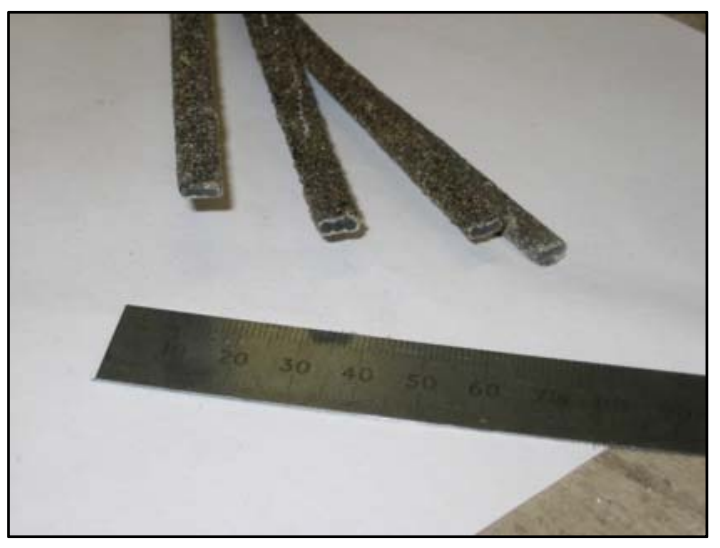

(b)

Figure 6: Examples of (a) BFRP and (b) CFRP bars used in the research 
Table 3: FRP properties

\begin{tabular}{|c|c|c|c|c|}
\hline FRP Type & $\begin{array}{c}\text { Average Cross } \\
\text { Sectional Area } \\
\left(\mathrm{mm}^{2}\right)\end{array}$ & $\begin{array}{l}\text { Average Tensile } \\
\text { Failure Load (kN) }\end{array}$ & $\begin{array}{l}\text { Rupture Strength } \\
\qquad\left(\mathrm{N} / \mathrm{mm}^{2}\right)\end{array}$ & $\begin{array}{l}\text { Elastic Modulus } \\
\qquad\left(\mathrm{N} / \mathrm{mm}^{2}\right)\end{array}$ \\
\hline CFRP & 19.65 & 19.46 & 990 & 77452 \\
\hline BFRP & 12.34 & 14.04 & 1138 & 35025 \\
\hline
\end{tabular}

\subsection{Test Slabs}

Idealised full size one-way spanning slabs were considered, as shown in Figure 7. For the purposes of evaluating in-plane restraint, only beams spanning parallel to the slab span were considered to offer a 'regular' restraint contribution, which is conservative compared to most bays within reinforced concrete frames. To facilitate laboratory testing, one-third scale test slabs were used and the following variables were investigated:

- Span-to-depth ratio, $(l / d)$.

- Level of in-plane restraint stiffness.

- Strengthening material.
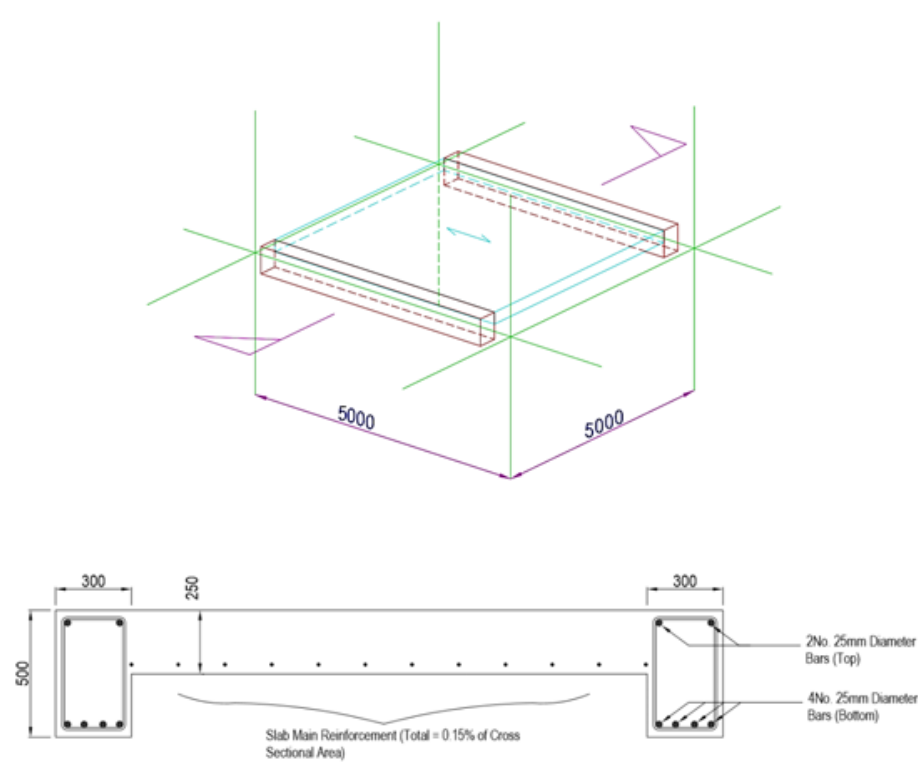

Figure 7: 'Regular' in-plane restraint arrangement in a typical building frame

\subsubsection{Span-to-depth ratio}

$l / d=20$ was chosen as being representative of typical building structure floor slabs.

For comparative purposes, slabs with $l / d=15$ were also tested, as this has been more commonly used in previous CMA studies. 


\subsubsection{Slab dimensions}

Seventeen test slabs were cast. Unrestrained samples were simply supported rectangular units whilst, in-plane restrained slabs incorporated parallel restraining beams, separated from the slab by a 50mm gap, as shown in Figures 8 and 9. Edge beam dimensions and reinforcement were then varied to achieve a range of restraint levels. Test slabs were coded to indicate restraint level, FRP and span-to-depth ratio as outlined below:
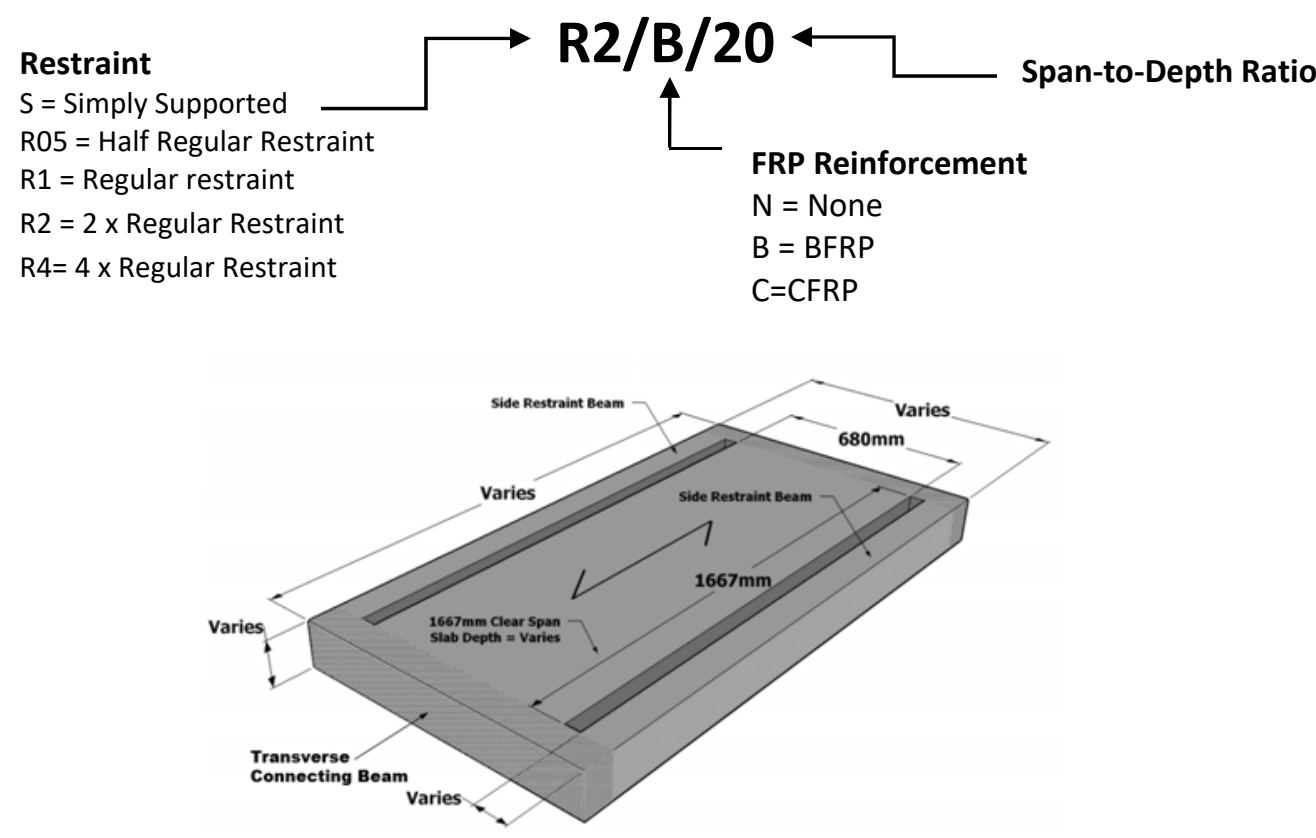

Figure 8: Typical Test Slab Geometry

Slab steel reinforcement was set slightly above minimum required design code levels at $0.15 \%$, as this was considered representative of many existing building structure floor slabs. All dimensions and reinforcement data are outlined in Figure 9 and Table 4 respectively.

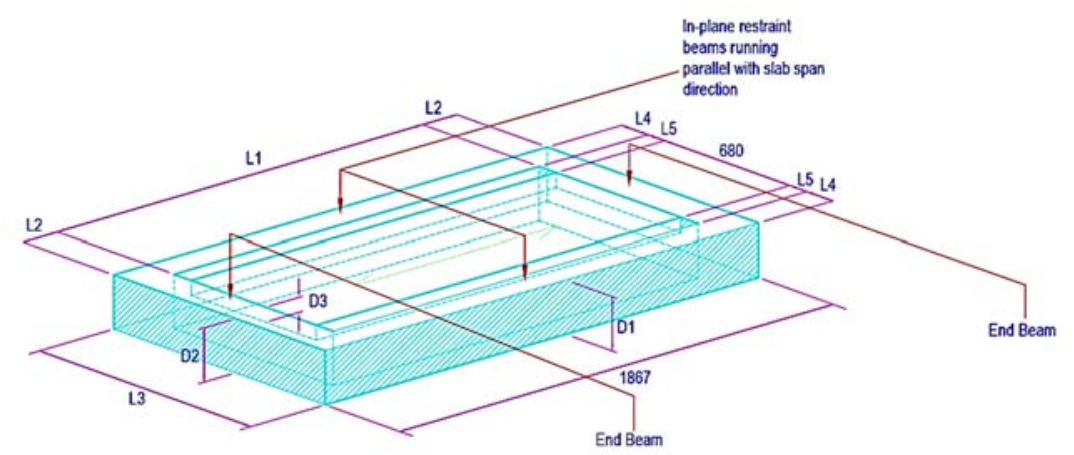

Figure 9: Typical In-plane restrained test slab (dimensions shown detailed in Table 4) 
Table 4: Slab dimensions and reinforcement

\begin{tabular}{|c|c|c|c|c|c|c|c|c|c|c|c|c|c|c|c|c|c|}
\hline \multirow[t]{2}{*}{ Slab Code } & \multirow{2}{*}{$\begin{array}{c}\text { Clear } \\
\text { Span } \\
\text { of } \\
\text { Slab, } \\
\text { L1 } \\
\\
(\mathrm{mm})\end{array}$} & \multirow{2}{*}{$\begin{array}{c}\begin{array}{c}\text { Width } \\
\text { of End } \\
\text { Beams, } \\
\text { L2 }\end{array} \\
(\mathrm{mm})\end{array}$} & \multirow{2}{*}{$\begin{array}{c}\begin{array}{c}\text { Total } \\
\text { Width } \\
\text { of } \\
\text { Sample, } \\
\text { L3 }\end{array} \\
\text { (mm) }\end{array}$} & \multirow{2}{*}{$\begin{array}{c}\text { Width of } \\
\text { In-plane } \\
\text { Restraint } \\
\text { Beams, } \\
\text { L4 } \\
\text { (mm) }\end{array}$} & \multirow{2}{*}{$\begin{array}{c}\text { Width of } \\
\text { Gap } \\
\text { Between } \\
\text { Slab and } \\
\text { In-plane } \\
\text { Restraint } \\
\text { Beams, } \\
\text { L5 } \\
\text { (mm) }\end{array}$} & \multirow{2}{*}{ 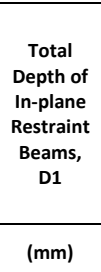 } & \multirow{2}{*}{$\begin{array}{l}\text { Total } \\
\text { Depth } \\
\text { of End } \\
\text { Beams } \\
\text {, D2 } \\
\text { (mm) }\end{array}$} & \multirow{2}{*}{$\begin{array}{c}\text { Total } \\
\text { Depth } \\
\text { of } \\
\text { Slab, } \\
\text { D3 } \\
\text { (mm) }\end{array}$} & \multicolumn{2}{|c|}{ Slab Steel Reinforcement } & \multirow{2}{*}{$\begin{array}{c}\begin{array}{c}\text { Percentage } \\
\text { Steel } \\
\text { Reinforcement } \\
\text { in Slab }\end{array} \\
\end{array}$} & \multirow{2}{*}{$\begin{array}{c}\begin{array}{c}\text { Effective Depth } \\
\text { of } \\
\text { Reinforcement } \\
\text { in Slab }\end{array} \\
(\mathrm{mm})\end{array}$} & \multicolumn{2}{|c|}{$\begin{array}{l}\text { Steel Reinforcement in } \\
\text { Parallel Beams }\end{array}$} & \multicolumn{2}{|c|}{$\begin{array}{l}\text { Steel Reinforcement in } \\
\text { End Beams }\end{array}$} & \multirow[t]{2}{*}{$\begin{array}{c}\text { Strengthenin } \\
\text { Material }\end{array}$} \\
\hline & & & & & & & & & & Bar Type & & & & Bar Type & & Bar Type & \\
\hline$S / N / 20$ & 1867 & 0 & 680 & 0 & 0 & 0 & 0 & 83.3 & 3 No. $6 \mathrm{~mm} \phi$ & Straights & 0.15 & 65.3 & None & NA & None & NA & N/A \\
\hline$S / N / 15$ & 1867 & 0 & 680 & 0 & 0 & 0 & 0 & 111.1 & 4 No. $6 \mathrm{~mm} \phi$ & Straights & 0.15 & 93.1 & None & NA & None & NA & N/A \\
\hline$s / c / 20$ & 1867 & 0 & 680 & 0 & 0 & 0 & 0 & 83.3 & 3 No. $6 \mathrm{~mm} \phi$ & Mesh & 0.15 & 65.3 & None & NA & None & NA & $\begin{array}{c}\text { 3No.CFRP @ } \\
19.65 \mathrm{~mm}^{2}\end{array}$ \\
\hline$S / B / 20$ & 1867 & 0 & 680 & 0 & 0 & 0 & 0 & 83.3 & $3 \mathrm{No} .6 \mathrm{~mm} \mathrm{\phi}$ & Straights & 0.15 & 65.3 & None & NA & None & NA & $\begin{array}{c}\text { 6No. BFRP @ } \\
12.34 \mathrm{~mm}^{2}\end{array}$ \\
\hline$S / B / 15$ & 1867 & 0 & 680 & 0 & 0 & 0 & 0 & 111.1 & 4No. $6 \mathrm{~mm} \phi$ & Straights & 0.15 & 93.1 & None & NA & None & NA & $\begin{array}{c}\text { 8No. BFRP @ } \\
12.34 \mathrm{~mm}^{2}\end{array}$ \\
\hline $\mathrm{R} 1 / \mathrm{N} / \mathbf{2 0}$ & 1667 & 100 & 920 & 70 & 50 & 100 & 100 & 83.3 & 3 No. $6 \mathrm{~mm} \phi$ & Straights & 0.15 & 65.3 & 4 No. $6 \mathrm{~mm} \phi$ & Straights & 4 No. $6 \mathrm{~mm} \phi$ & Straights & N/A \\
\hline $\mathrm{R} 0.5 / \mathrm{N} / 20$ & 1667 & 100 & 880 & 50 & 50 & 60 & 83.3 & 83.3 & 3 No. $6 \mathrm{~mm} \phi$ & Straights & 0.15 & 65.3 & 4 No. $6 \mathrm{~mm} \phi$ & Straights & $4 \mathrm{No} .6 \mathrm{~mm} \mathrm{\phi}$ & Straights & N/A \\
\hline $\mathrm{R} 2 / \mathrm{N} / 20$ & 1667 & 100 & 980 & 100 & 50 & 147 & 147 & 83.3 & 3 No. $6 \mathrm{~mm} \phi$ & Straights & 0.15 & 65.3 & $4 \mathrm{No} .6 \mathrm{~mm} \mathrm{\phi}$ & Straights & $4 \mathrm{No} .6 \mathrm{~mm} \mathrm{\phi}$ & Straights & N/A \\
\hline $\mathrm{R} 2 / \mathrm{N} / 15$ & 1667 & 100 & 980 & 100 & 50 & 143 & 143 & 111.1 & 4 No. $6 \mathrm{~mm} \phi$ & Straights & 0.15 & 93.1 & $4 \mathrm{No} .6 \mathrm{~mm} \phi$ & Straights & $4 \mathrm{No} .6 \mathrm{~mm} \phi$ & Straights & N/A \\
\hline $\mathrm{R} 4 / \mathrm{N} / 15$ & 1667 & 100 & 980 & 100 & 50 & 143 & 143 & 111.1 & $4 \mathrm{No} .6 \mathrm{~mm} \mathrm{\phi}$ & Mesh & 0.15 & 93.1 & 2No. $32 \mathrm{~mm} \phi$ & Bent Bars & $\begin{array}{c}2 \text { 2No. } 32 \mathrm{~mm} \\
\phi\end{array}$ & Bent Bars & N/A \\
\hline $\mathrm{R} 1 / \mathrm{C} / 20$ & 1667 & 100 & 920 & 70 & 50 & 100 & 100 & 83.3 & 3No. $6 \mathrm{~mm} \mathrm{\phi}$ & Mesh & 0.15 & 65.3 & 4No. $6 \mathrm{~mm} \phi$ & Mesh & 4 No. $6 \mathrm{~mm} \phi$ & Mesh & $\begin{array}{c}\text { 3No.CFRP @ } \\
19.65 \mathrm{~mm}^{2}\end{array}$ \\
\hline $\mathrm{R} 1 / \mathrm{B} / 20$ & 1667 & 100 & 920 & 70 & 50 & 100 & 100 & 83.3 & 3 No. $6 \mathrm{~mm} \phi$ & Mesh & 0.15 & 65.3 & 4No. $6 \mathrm{~mm} \mathrm{\phi}$ & Mesh & 4No. $6 \mathrm{~mm} \mathrm{\phi}$ & Mesh & $\begin{array}{c}\text { 6No. BFRP @ } \\
12.34 \mathrm{~mm}^{2}\end{array}$ \\
\hline $\mathrm{R} 0.5 / \mathrm{C} / 20$ & 1667 & 100 & 880 & 50 & 50 & 60 & 83.3 & 83.3 & 3No. $6 \mathrm{~mm} \mathrm{\phi}$ & Mesh & 0.15 & 65.3 & 4No. $6 \mathrm{~mm} \mathrm{\phi}$ & Mesh & 4No. $6 \mathrm{~mm} \mathrm{\phi}$ & Mesh & $\begin{array}{c}\text { 3No. CFRP @ } \\
19.65 \mathrm{~mm}^{2}\end{array}$ \\
\hline R0.5/B/20 & 1667 & 100 & 880 & 50 & 50 & 60 & 83.3 & 83.3 & 3No. $6 \mathrm{~mm} \phi$ & Straights & 0.15 & 65.3 & 4No. $6 \mathrm{~mm} \mathrm{\phi}$ & Straights & 4 No. $6 \mathrm{~mm} \phi$ & Straights & $\begin{array}{c}\text { 6No. BFRP @ } \\
12.34 \mathrm{~mm}^{2} \\
\end{array}$ \\
\hline $\mathrm{R} 2 / \mathrm{C} / 20$ & 1667 & 100 & 980 & 100 & 50 & 147 & 147 & 83.3 & $3 \mathrm{No} .6 \mathrm{~mm} \mathrm{\phi}$ & Mesh & 0.15 & 65.3 & 4 No. $6 \mathrm{~mm} \mathrm{\phi}$ & Mesh & 4 No. $6 \mathrm{~mm} \phi$ & Mesh & $\begin{array}{c}\text { 3No.CFRP @ } \\
19.65 \mathrm{~mm}^{2}\end{array}$ \\
\hline $\mathrm{R} 2 / \mathrm{B} / 20$ & 1667 & 100 & 980 & 100 & 50 & 147 & 147 & 83.3 & 3 No. $6 \mathrm{~mm} \phi$ & Straights & 0.15 & 65.3 & 4No. $6 \mathrm{~mm} \phi$ & Straights & 4 No. $6 \mathrm{~mm} \phi$ & Straights & $\begin{array}{c}\text { 6No. BFRP @ } \\
12.34 \mathrm{~mm}^{2} \\
\end{array}$ \\
\hline $\mathrm{R} 2 / \mathrm{B} / 15$ & 1667 & 100 & 980 & 100 & 50 & 143 & 143 & 111.1 & 4No. $6 \mathrm{~mm} \phi$ & Mesh & 0.15 & 93.1 & 4 No. $6 \mathrm{~mm} \mathrm{\phi}$ & Mesh & $4 \mathrm{NN} .6 \mathrm{~mm} \mathrm{\phi}$ & Mesh & $\begin{array}{c}\text { 8No. BFRP@ } \\
12.34 \mathrm{~mm}^{2} \\
\end{array}$ \\
\hline
\end{tabular}




\subsubsection{Test slab concrete strengths}

On the day of each slab test, compressive and tensile strengths for each slab were established as discussed in section 2.1.1. These are summarised in Table 5.

Table 5: Test slab concrete properties

\begin{tabular}{|c|c|c|c|c|}
\hline $\begin{array}{l}\text { Slab } \\
\text { Code }\end{array}$ & Workability & $\begin{array}{c}\text { Slump } \\
(\mathrm{mm})\end{array}$ & $\begin{array}{c}\text { Compressive Cube Strength } \\
\mathrm{f}_{\mathrm{ck}, \text { cube }}\left(\mathrm{N} / \mathrm{mm}^{2}\right)\end{array}$ & $\begin{array}{c}\text { Tensile Strength } \mathrm{f}_{\mathrm{ct}} \\
\left(\mathrm{N} / \mathrm{mm}^{2}\right)\end{array}$ \\
\hline S/N/20 & "Satisfactory & 25 & 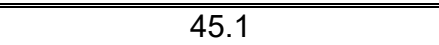 & 3.0 \\
\hline S/N/15 & Satisfactory & 35 & 51.8 & 3.3 \\
\hline $\mathrm{S} / \mathrm{C} / 20$ & Satisfactory & 10 & 44.3 & 3.0 \\
\hline S/B/20 & Satisfactory & 35 & 60.1 & 3.7 \\
\hline S/B/15 & Satisfactory & 35 & 45.5 & 3.1 \\
\hline R1/N/20 & Satisfactory & 35 & 45.1 & 3.0 \\
\hline R05/N/20 & Satisfactory & 30 & 46.0 & 3.1 \\
\hline R2/N/20 & Satisfactory & 30 & 45.5 & 3.1 \\
\hline R2/N/15 & Satisfactory & 35 & 45.1 & 3.0 \\
\hline R4/N/15 & Satisfactory & 40 & 39.1 & 2.8 \\
\hline $\mathrm{R} 1 / \mathrm{C} / 20$ & Satisfactory & 40 & 43.1 & 3.0 \\
\hline $\mathrm{R} 1 / \mathrm{B} / 20$ & Satisfactory & 35 & 50.9 & 3.3 \\
\hline $\mathrm{R} 05 / \mathrm{C} / 20$ & Satisfactory & 35 & 54.0 & 3.4 \\
\hline R05/B/20 & Satisfactory & 35 & 52.7 & 3.4 \\
\hline $\mathrm{R} 2 / \mathrm{C} / 20$ & Satisfactory & 40 & 43.1 & 3.0 \\
\hline $\mathrm{R} 2 / \mathrm{B} / 20$ & Satisfactory & 35 & 52.7 & 3.4 \\
\hline R2/B/15 & Satisfactory & 45 & 43.3 & 3.0 \\
\hline
\end{tabular}

\subsubsection{Slab test instrumentation}

A typical slab test arrangement is illustrated in Figure 10. All slabs were supported on roller supports. For restrained samples, electrical resistance strain (ERS) gauges were attached to the upper and lower faces of each steel reinforcement bar within one restraint beam at mid-span.

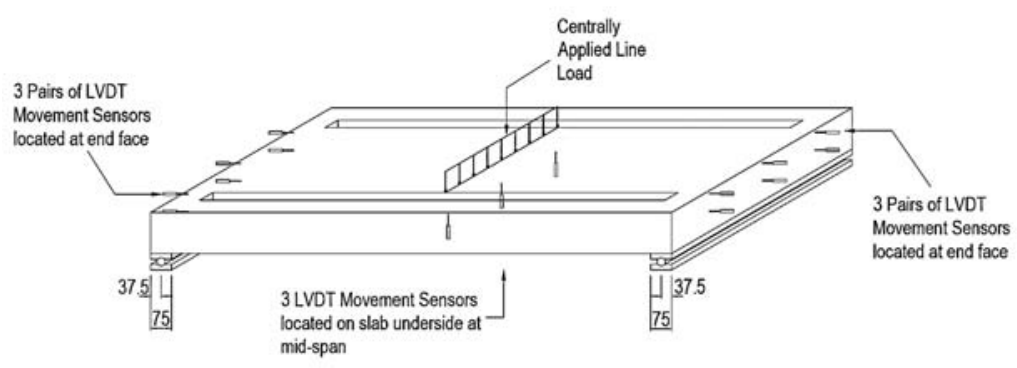

Figure 10: Typical test slab setup 


\subsubsection{Level of in-plane restraint stiffness}

As the degree of in-plane restraint affects the level of compressive membrane action, stiffness ratios of zero (i.e. simply supported), 'regular', '0.5x regular', ' $2 x$ regular' and ' $4 x$ regular' were used, where 'regular' restraint was considered representative of that experienced by a typical floor slab (i.e. R1/N/20) shown in Figure 7 with details outlined in Table 6.

Table 6: 'Regular' restrained slab details

\begin{tabular}{|c|c|}
\hline Elastic modulus of steel, $\mathbf{E}_{\mathbf{s}}$ & $210000 \mathrm{~N} / \mathrm{mm}^{2}$ \\
\hline Elastic modulus of concrete, $\mathbf{E}_{\mathbf{c}}$ & $26753 \mathrm{~N} / \mathrm{mm}^{2}$ \\
\hline Tie Beam Width & $300 \mathrm{~mm}$ \\
\hline Tie Beam Depth & $500 \mathrm{~mm}$ \\
\hline Total Tie Beam Reinforcement & 6No. 25mm Diameter Bars \\
\hline Slab Width & $5000 \mathrm{~mm}$ \\
\hline Slab Depth & $250 \mathrm{~mm}$ \\
\hline Total Slab Reinforcement & $0.15 \%$ Slab Cross Sectional Area \\
\hline
\end{tabular}

In this case, $E_{c}$ was assumed to be directly related to compressive cube strength, $f_{c k, c u b e}$, using the Hognestad [55] relationship:

$$
E_{c}=4230 f_{c k, c u b e^{1 / 2}}
$$

Based on the 'regular' restrained slab shown in Figure 7; and using data in Table 6; slab stiffness $k_{s}$, restraint stiffness $k_{r}$ and stiffness ratio $r$ were evaluated for a 'regular' level of in-plane restraint to give:

$k_{S}=k_{\text {slab }}=\frac{E_{c, \text { Slab }} A_{c, \text { Slab }}}{L_{\text {Slab }}}+\frac{E_{S, \text { Slab }} A_{S, \text { Slab }}}{L_{\text {Slab }}}+\frac{E_{F R P} A_{F R P}}{L_{\text {Slab }}}=6756968 \mathrm{~N} / \mathrm{mm}$

$k_{r}=2 \times k_{\text {Beam }}=2 \times\left\{\frac{E_{c, \text { Beam }} A_{c, \text { Beam }}}{L_{\text {Beam }}}+\frac{E_{s, \text { Beam }} A_{S, \text { Beam }}}{L_{\text {Beam }}}\right\}=1821063 \mathrm{~N} / \mathrm{mm}$

$r=\frac{k_{r}}{k_{s}}=\frac{1821063}{6756968}=0.270$

\subsubsection{Preliminary in-plane restraint stiffness}

Prior to considering an analysis of experimental readings from each test slab, it was possible to estimate the in-plane restraint stiffness, $k_{r}$, and slab stiffness, $k_{s}$, by 
assuming simplified purely axial behaviour. These estimates were considered with either 'half cracked' or 'uncracked' restraint beam behaviour for later comparison with more exact experimental values. The 'uncracked' condition considered restraint beam concrete having its full elastic modulus, $E_{c}$, value whilst the 'half cracked' condition considered $50 \%$ of the $E_{c}$ value to simulate the presence of beam cracking and the resulting reduction in effective concrete cross sectional area. Both cases included reinforcement. Preliminary $k_{r}$ estimates are shown in Table 7 along with corresponding preliminary stiffness ratio estimates, $r$.

Table 7: Preliminary restraint and stiffness estimates

\begin{tabular}{|c|c|c|c|c|c|c|}
\hline \multirow{2}{*}{$\begin{array}{l}\text { Slab } \\
\text { Code }\end{array}$} & \multicolumn{2}{|c|}{$\begin{array}{l}\text { Preliminary Restraint } \\
\text { Stiffness, } k_{r}(k N / m m)\end{array}$} & \multicolumn{2}{|c|}{$\begin{array}{c}\text { Preliminary Slab } \\
\text { Stiffness, ks (kN/mm) }\end{array}$} & \multicolumn{2}{|c|}{$\begin{array}{c}\text { Preliminary Stiffness } \\
\text { Ratio, } r\end{array}$} \\
\hline & $\begin{array}{c}\text { Half } \\
\text { cracked }\end{array}$ & Uncracked & $\begin{array}{c}\text { Half } \\
\text { cracked }\end{array}$ & Uncracked & $\begin{array}{c}\text { Half } \\
\text { cracked }\end{array}$ & Uncracked \\
\hline R1/N/20 & 135.10 & 244.25 & 4457.96 & 906.19 & 0.29 & 0.27 \\
\hline R0.5/N/20 & 72.16 & 118.38 & 462.48 & 915.23 & 0.16 & 0.13 \\
\hline $\mathrm{R} 2 / \mathrm{N} / 20$ & 258.26 & 490.58 & 460.13 & 910.52 & 0.56 & 0.54 \\
\hline R2/N/15 & 250.74 & 475.55 & 610.63 & 1208.30 & 0.41 & 0.39 \\
\hline R4/N/15 & 523.83 & 711.07 & 568.27 & 1124.71 & 0.92 & 0.63 \\
\hline $\mathrm{R} 1 / \mathrm{C} / 20$ & 130.42 & 237.18 & 449.79 & 888.15 & 0.29 & 0.27 \\
\hline $\mathrm{R} 1 / \mathrm{B} / 20$ & 139.62 & 255.56 & 486.44 & 962.55 & 0.29 & 0.27 \\
\hline $\mathrm{R} 0.5 / \mathrm{C} / 20$ & 73.75 & 123.83 & 502.00 & 992.58 & 0.15 & 0.12 \\
\hline $\mathrm{R} 0.5 / \mathrm{B} / 20$ & 75.39 & 124.83 & 495.55 & 979.92 & 0.15 & 0.13 \\
\hline $\mathrm{R} 2 / \mathrm{C} / 20$ & 249.76 & 475.86 & 449.75 & 888.08 & 0.56 & 0.54 \\
\hline $\mathrm{R} 2 / \mathrm{B} / 20$ & 275.80 & 525.67 & 495.58 & 979.99 & 0.56 & 0.54 \\
\hline R2/B/15 & 243.96 & 464.26 & 599.45 & 1185.13 & 0.41 & 0.39 \\
\hline
\end{tabular}

\subsubsection{Concrete stresses within restraint beams}

\section{Compressive concrete stresses}

Concrete compressive stresses were related to plane strains using the Thorenfeldt et al. [56] constitutive relationship, as defined in equations (27-31) and illustrated in Figure 11. 


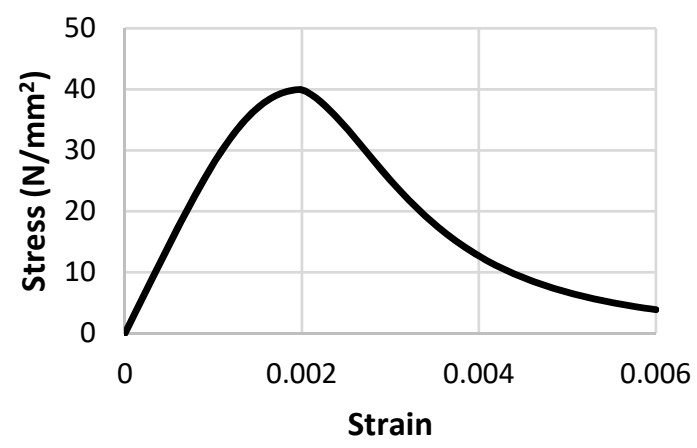

Figure 11: Compression constitutive relationship for concrete with $f_{\text {ck,cube }}=40 \mathrm{~N} / \mathrm{mm}^{2}[56]$

$$
\begin{aligned}
& \sigma_{c}=f_{c k, \text { cube }} \frac{n\left[\frac{\varepsilon_{c}}{\varepsilon_{0}}\right]}{n-1+\left[\frac{\varepsilon_{c}}{\varepsilon_{0}}\right]^{n k}} \\
& n=\frac{E_{c i}}{E_{c i}-E_{\text {secant }}} \\
& E_{c i}=4230 \sqrt{f_{\text {ck,cube }}} \quad \text { (Hognestad, [55]) } \\
& E_{\text {secant }}=\frac{f_{\text {ck,cube }}}{\varepsilon_{0}}
\end{aligned}
$$

$k=1$ for $\varepsilon_{c} \leq \varepsilon_{0}$

$$
=0.67+\left[\frac{f_{c k, c u b e}}{77.5}\right] \text { for } \varepsilon_{c}>\varepsilon_{0} \quad \text { The value of } \mathrm{k} \text { must not be greater than } 1
$$

$$
\text { where: } \quad \begin{aligned}
& \sigma_{c}=\text { Compressive stress }\left(\mathrm{N} / \mathrm{mm}^{2}\right) \\
& \varepsilon_{c}=\text { Compressive strain } \\
& \varepsilon_{0}=\text { Compressive strain corresponding to } f_{c k, c u b e} \\
& E_{c i}=\text { Initial elastic modulus of concrete }\left(\mathrm{N} / \mathrm{mm}^{2}\right) \\
& E_{\text {secant }}=\text { Secant modulus of concrete }\left(\mathrm{N} / \mathrm{mm}^{2}\right) \\
& f_{c k, \text { cube }}=\text { Concrete compressive cube strength }\left(\mathrm{N} / \mathrm{mm}^{2}\right) \\
& n=\text { Curve fitting factor } \\
& k=\text { post peak decay term }
\end{aligned}
$$

\section{Tensile Concrete Stresses}

Concrete tensile stresses were established using a bilinear constitutive relationship [57] based on linearly interpolated plane strain readings from beam reinforcement bars, as shown in Figure 12 and described in equation (32). This considered concrete as having no tensile capability beyond the ultimate tensile strain limit, $\varepsilon_{u l t}$. A 
recommended value for $\varepsilon_{u l t}$ has previously been defined as $2.5 \times 10^{-3}$ [58] which was adopted in the research.

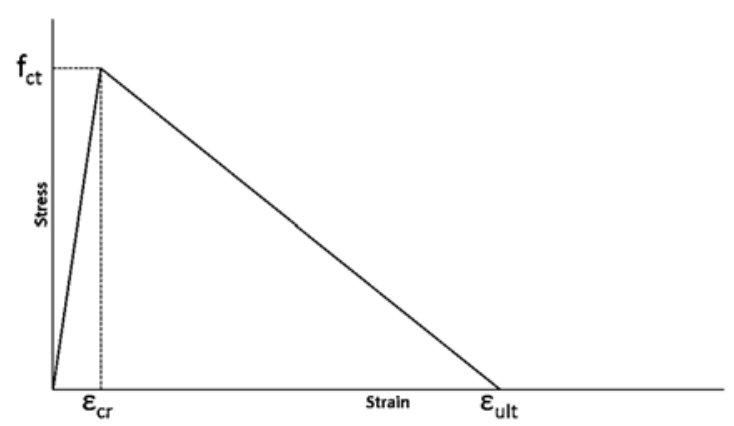

Figure 12: Tensile stress-strain behaviour of concrete with linear softening [57]

$$
\begin{array}{rlrl}
\sigma_{t} & =E_{c} \varepsilon_{t} & 0<\varepsilon_{t} \leq \varepsilon_{c r} \\
& =f_{c t}\left[1-\frac{\left(\varepsilon_{t}-\varepsilon_{c r}\right)}{\left(\varepsilon_{u l t}-\varepsilon_{c r}\right)}\right] & \varepsilon_{c r}<\varepsilon_{t} \leq \varepsilon_{u l t} \\
& =0 & \varepsilon_{t}>\varepsilon_{u l t}
\end{array}
$$

where: $\quad \sigma_{t}=$ Tensile stress $\left(\mathrm{N} / \mathrm{mm}^{2}\right)$

$\varepsilon_{t}=$ Tensile strain

$E_{c}=$ Elastic modulus of concrete $\left(\mathrm{N} / \mathrm{mm}^{2}\right)$

$f_{c t}=$ Tensile strength of concrete $\left(\mathrm{N} / \mathrm{mm}^{2}\right)$

$\varepsilon_{c r}=$ Critical tensile strain in concrete corresponding with $f_{c t}$

$\varepsilon_{u l t}=$ Ultimate tensile strain in concrete

$$
\begin{aligned}
& E_{c}=4230 \sqrt{f_{c k, c u b e}} \quad \text { (Hognestad, [55]) } \\
& \varepsilon_{c r}=\frac{f_{c t}}{E_{c}}
\end{aligned}
$$

\subsubsection{Restraint beam forces and bending moments}

From the experimental strain distribution, and the resulting stress-strain relationships, a stress distribution was determined. This was then integrated over the depth of each restraint beam section to determine resultant axial loads and bending moments.

\subsubsection{Transverse connecting beam torsion capacity}

Restrained test slabs experienced some fixity due to transverse connecting beam torsional capacity and the inclusion of this component of moment resistance was 
included in the overall evaluation of system stiffness [59] with the omission of material and safety factors.

\subsubsection{Evaluation of experimental restraint stiffness}

Bending and axial effects within restraint beams, along with the torsional capacity of transverse connecting beams, were equated to an equivalent compressive force which was considered to act at the mid-depth of each test slab. Corresponding slab mid-depth extensions were obtained from interpolated end face extension measurements and the ratio of equivalent compressive force to slab mid-depth extension was determined as an 'equivalent restraint stiffness'.

The result of evaluating the overall in-plane restraint stiffness in this way was to effectively consider the slabs as separate to their surrounding restraint system; reacting against an equivalent, purely compressive force at mid-depth, where corresponding extensions were also evaluated, as shown in Figure 13. This consideration therefore overcame difficulties associated with differing slab and restraint beam depths and the eccentricities between their resultant internal forces.

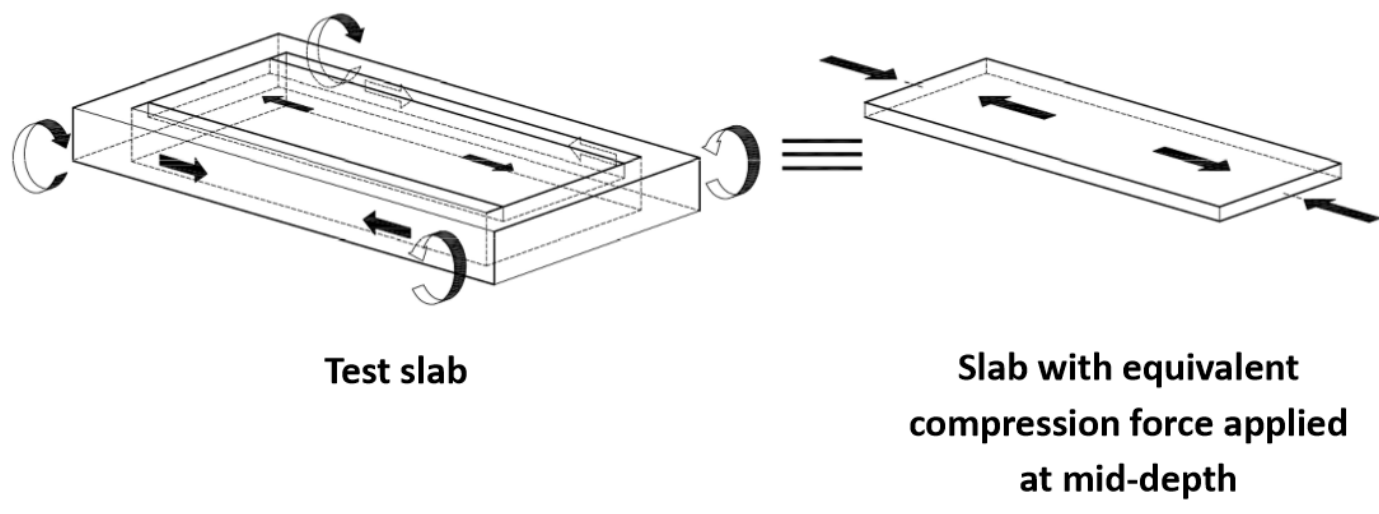

Figure 13: Variation of equivalent slab compression with extension at middepth

\section{Results}

\subsection{Test slab behaviour}

Investigations of extension with equivalent compressive force at mid-depth in restrained test slabs showed that restraint stiffness varied as loading increased. This was due to a combination of cracking within restraint beams, within slabs and at transverse connecting-beam-to-restraint-beam corners. Common trends in the restraint stiffness variation were also observed within test subgroups. Due to the very 
small movements involved; and very low strains within restraint beam reinforcement at initial stages of loading; the sensitivity of LVDTs $( \pm 0.01 \mathrm{~mm})$ and ERS gauges $\left( \pm 0.85 \mu \mathrm{m} / \mathrm{m} /{ }^{0} \mathrm{C}\right)$; only equivalent experimental restraint stiffness values which became significant in the latter stages of loading have been illustrated in graphical output (see Figures 15, 17, 19, 21 and 22) for these investigations. This is primarily because only the external in-plane restraint at peak load is required for predicting arching capacity and also because CMA develops only after slab cracking occurs. Indeed, at very low applied loads, compressive forces were established from ERS gauges readings before many LVDTs recorded any extension; resulting in theoretically infinite stiffness, which was of no practical benefit within the research. Hence, recordings were only considered of practical significance after samples had sufficiently 'bedded in'.

For shallow slabs with low restraint, equivalent compression variation with extension at mid-depth trends, as shown in Figure 14, were similar up to peak stiffness. Reductions in stiffness were due to simultaneous rising extension and falling compression. Figure 15 compares equivalent restraint stiffnesses with preliminary axial estimates and shows that $\mathrm{R} 05 / \mathrm{N} / 20$ and $\mathrm{R} 05 / \mathrm{C} / 20$ were approximately two thirds between 'reinforcement only' and 'half cracked' levels at failure. R05/B/20 displayed restraint levels indicative of only beam reinforcement acting in tension, with no contribution from concrete due to concrete 'honeycombing' in this particular test sample. The presence of concrete honeycombing in this test slab is therefore considered responsible for a reduction in both flexural and arching behaviour.

Equivalent compression variation with extension at mid-depth trends for shallow slabs with regular restraint are shown in Figure 16. Similar trends occurred in these samples up to initial slab cracking. Approximately linear behaviour then occurred in all cases with differing gradients. However, R1/B/20 displayed a sudden extension reduction due to beam cracking. Ultimate failure in each case was due to full torsional corner cracking. Figure 17 shows how equivalent restraint stiffnesses decreased at similar rates in these samples. With reference to axial preliminary estimates, R1/N/20 was approximately equal to 'uncracked' stiffness and R1/B/20 was $38 \%$ higher than 'uncracked' stiffness at failure. R1/C/20, however, had a final stiffness one third between 'half cracked' and 'uncracked' levels at failure.

Shallow slabs with high levels of restraint all displayed similar and significant increases in equivalent compressive force with very small increases in mid-depth extension, as shown in Figure 18. Beyond this initial region, R2/N20 and R2/B/20 
displayed similar growth in equivalent compressive force compared with R2/C/20. All three samples experienced their highest levels of equivalent compressive force and mid-depth extension at failure, when torsional corner cracks fully developed. Reduction of equivalent restraint stiffness was similar in $R 2 / B / 20$ and $R 2 / C / 20$ compared with $R 2 / N / 20$, as shown in Figure 19. It was also notable that a rapid reduction in restraint stiffness occurred in $\mathrm{R} 2 / \mathrm{C} / 20$ when the first end support upper surface crack appeared. However, both $\mathrm{R} 2 / \mathrm{N} / 20$ and R2/B/20 had final equivalent stiffnesses approximately halfway between the purely axial 'half cracked' and 'uncracked' estimates. R2/C/20 had a final equivalent stiffness slightly below the 'half cracked' estimate.

For deep slabs with high restraint, R2/B/15 displayed much lower extension at similar initial loads compared with $R 2 / N / 15$, as shown in Figure 20 . It is notable that $R 2 / B / 15$ displayed a step in extension from $0.003 \mathrm{~mm}$ at $48.08 \mathrm{kN}$ to $-0.035 \mathrm{~mm}$ at $51.18 \mathrm{kN}$. This was due to higher inward movements in upper end face LVDTs compared with the outward movements of lower end face LVDTs and the interpolative relationship between these values in establishing slab mid-depth movement. However, a review of the data has shown that this occurred prior to any observable crack development in both slab and beam regions. Reductions in equivalent restraint stiffness occurred in these cases due to torsional corner cracking and final equivalent stiffnesses were between purely axial 'half cracked' and 'reinforcement only' estimates, as shown in Figure 21. Samples R2/N/15 and R4/N/15 displayed similar equivalent force versus extension at mid-depth behaviour up to the point where slab cracking occurred in R4/N/15, as shown in Figure 20. However, for intermediate loads, extensions were higher in R4/N/15 before this trend was reversed at higher loads ( $>150 \mathrm{kN}$ ). Figure 22 shows that final equivalent restraint stiffness at failure, for $\mathrm{R} 4 / \mathrm{N} / 15$ was approximately two thirds between 'reinforcement only' and 'half cracked' estimates. 


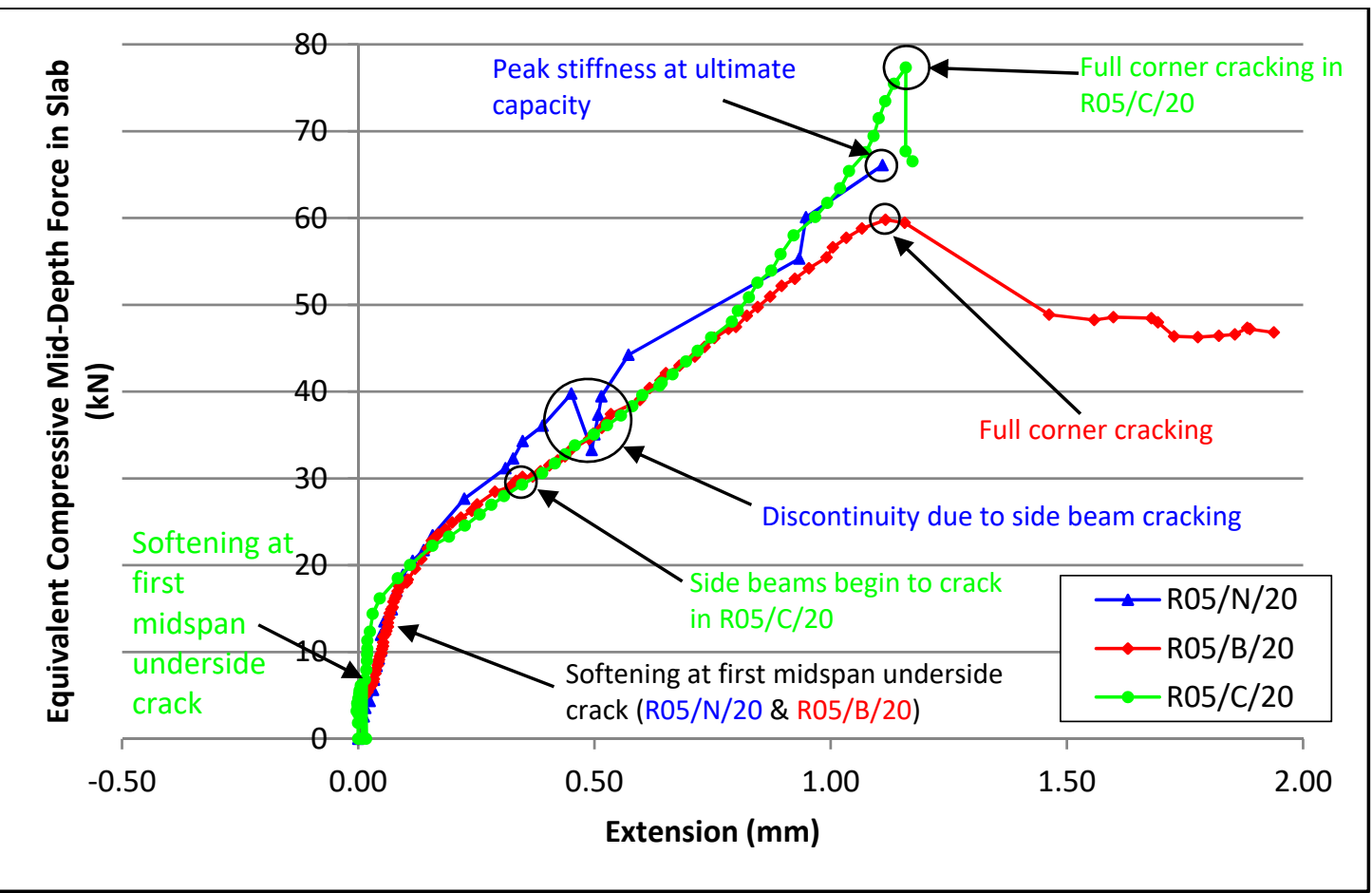

Figure 14: Variation of equivalent mid-depth slab compression with extension for shallow slabs with low restraint

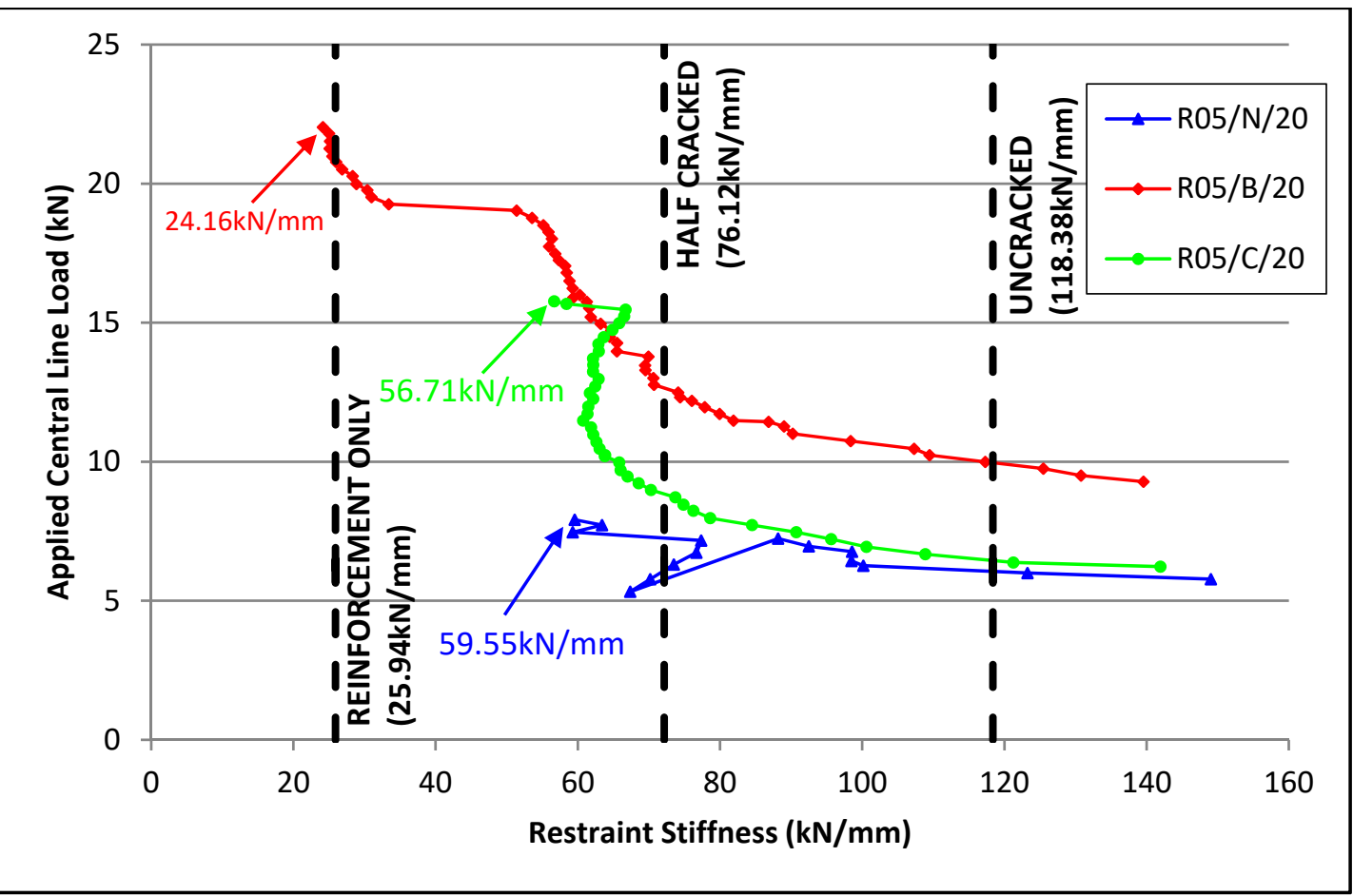

Figure 15: Variation of equivalent restraint stiffness with applied load for shallow slabs with low restraint 


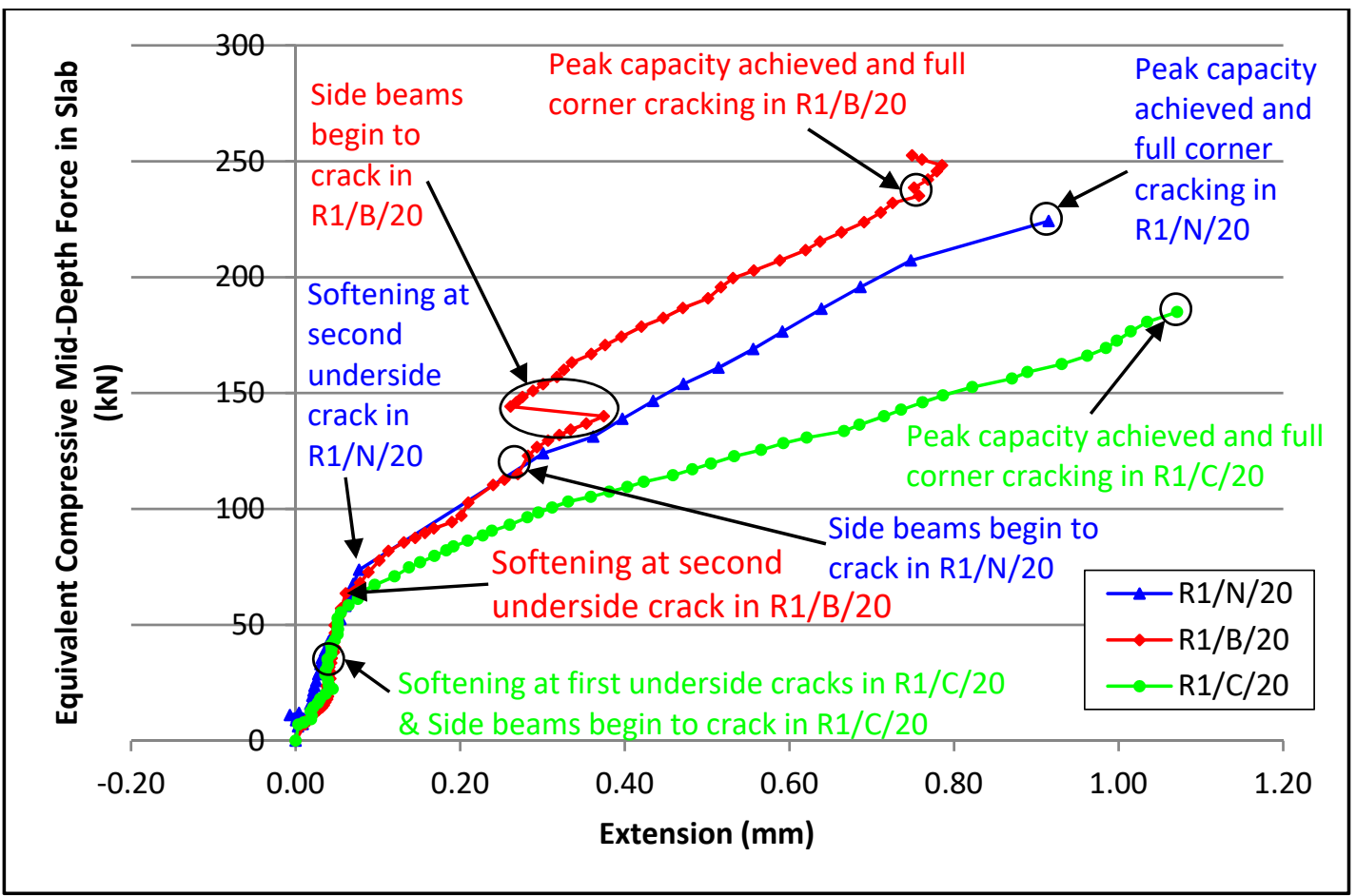

Figure 16: Variation of equivalent mid-depth slab compression with extension for shallow slabs with regular restraint

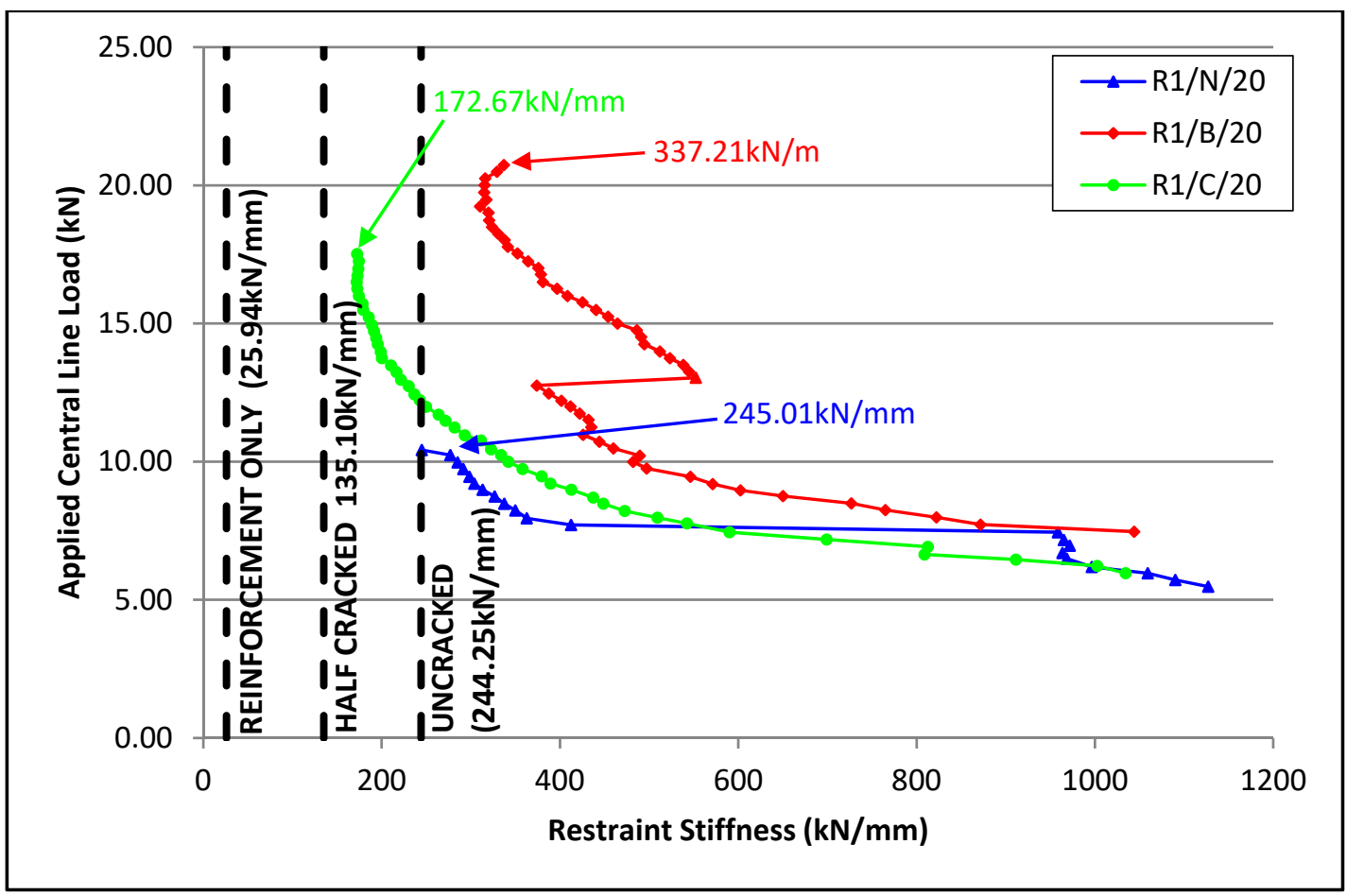

Figure 17: Variation of equivalent restraint stiffness with applied load for shallow slabs with regular restraint 


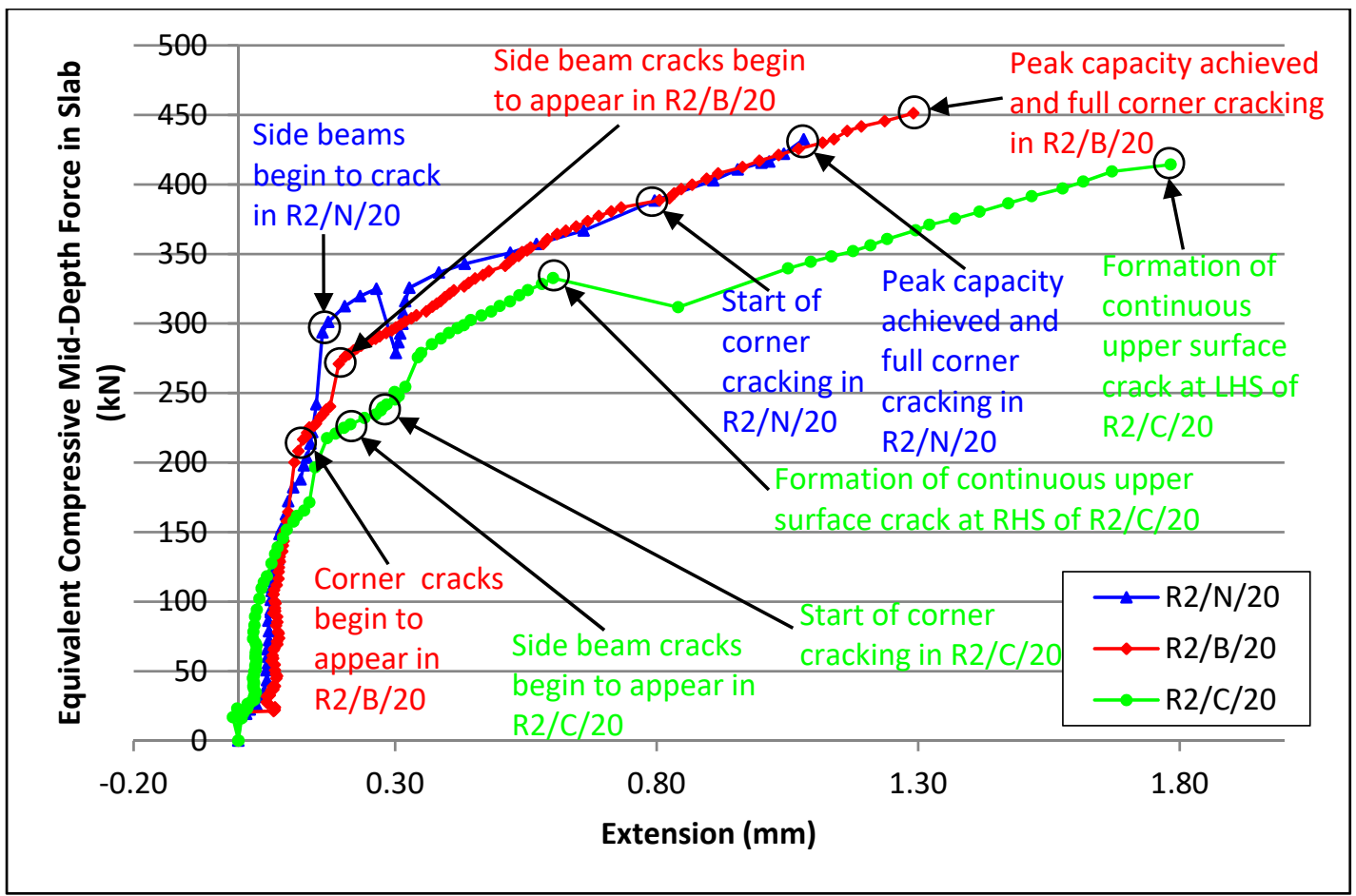

Figure 18: Variation of equivalent mid-depth slab compression with extension for shallow slabs with high restraint

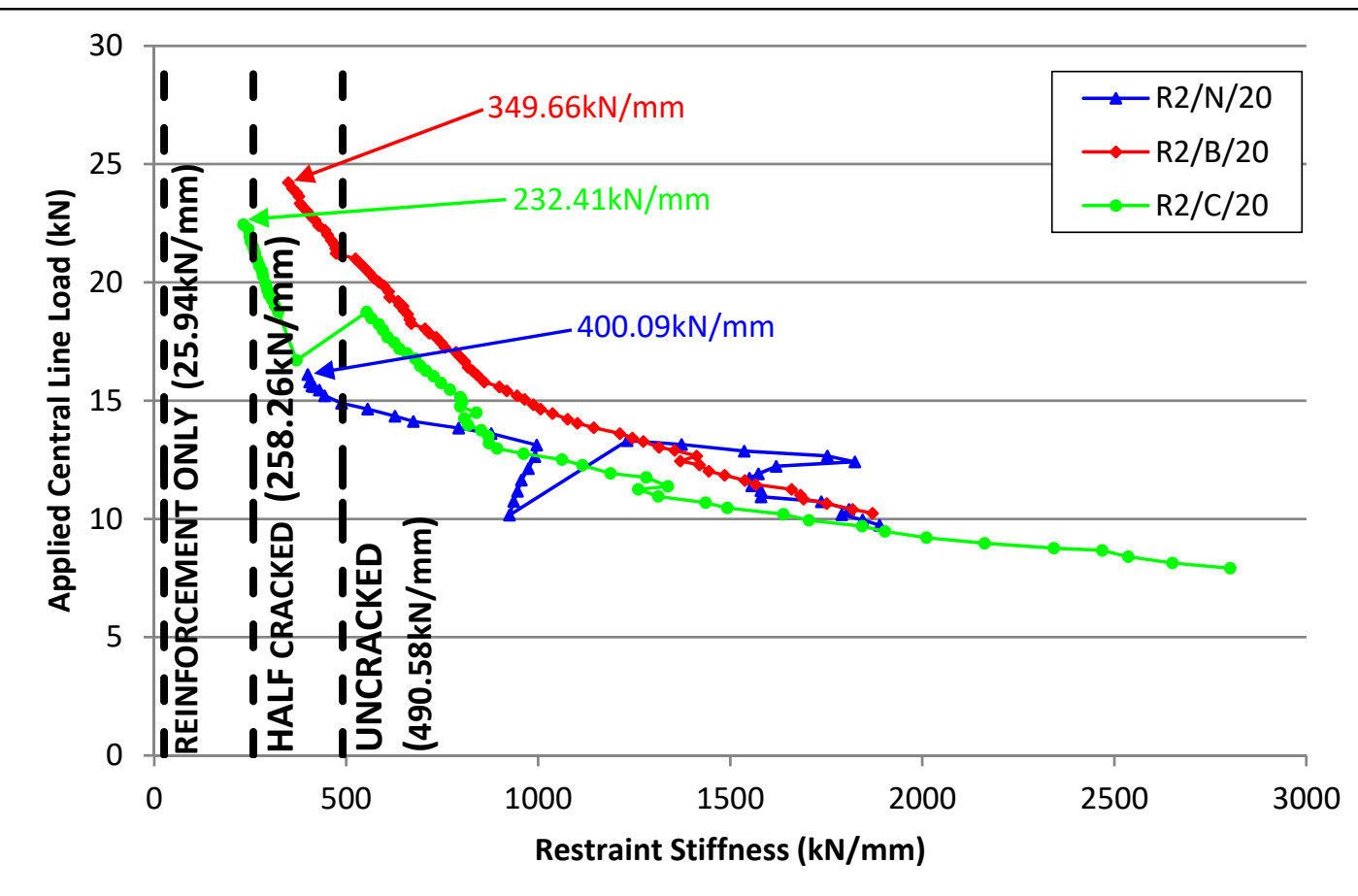

Figure 19: Variation of equivalent restraint stiffness with applied load for shallow slabs with high restraint 


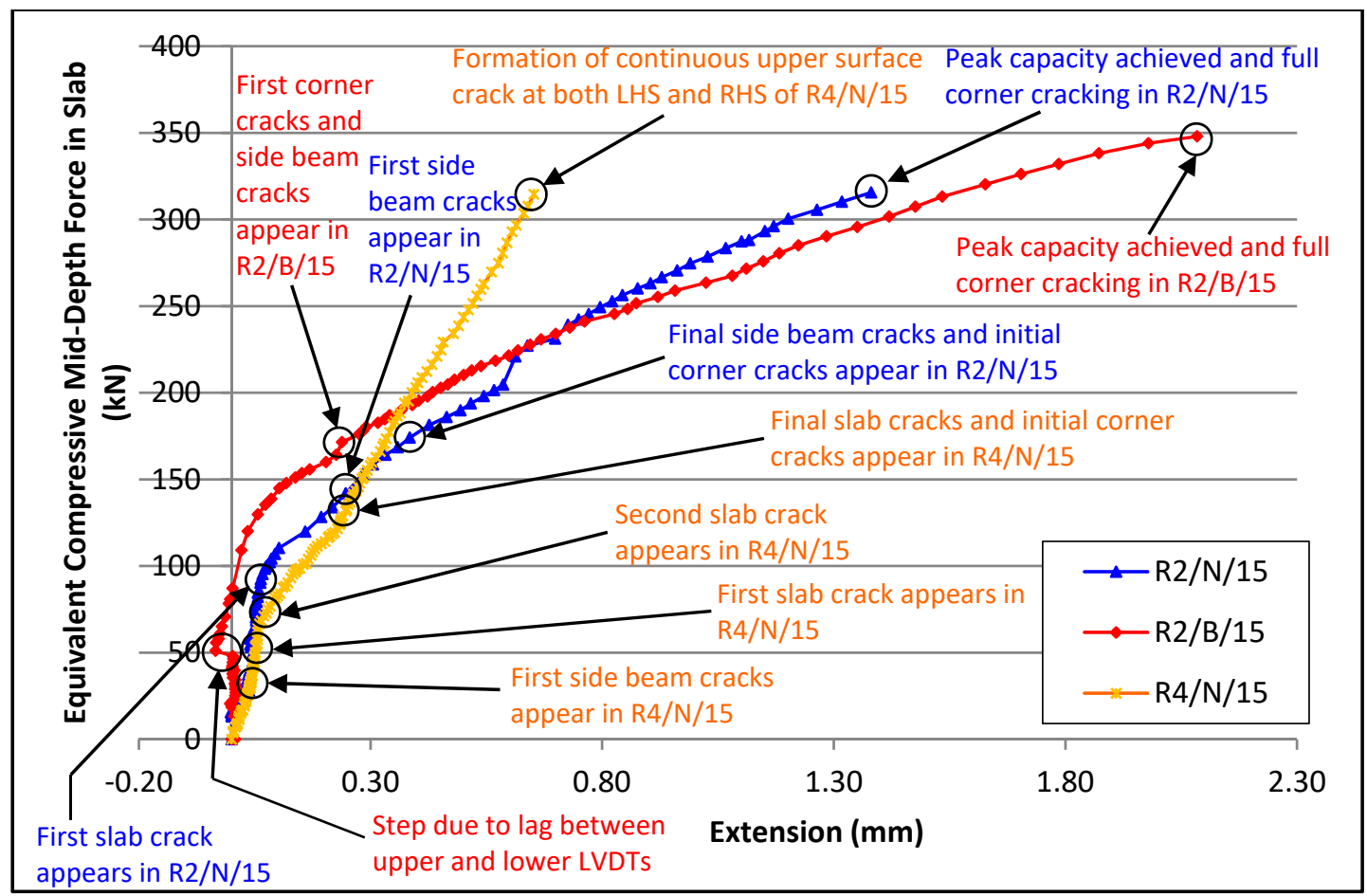

Figure 20: Variation of equivalent mid-depth slab compression with extension for deep slabs with high and very high restraint

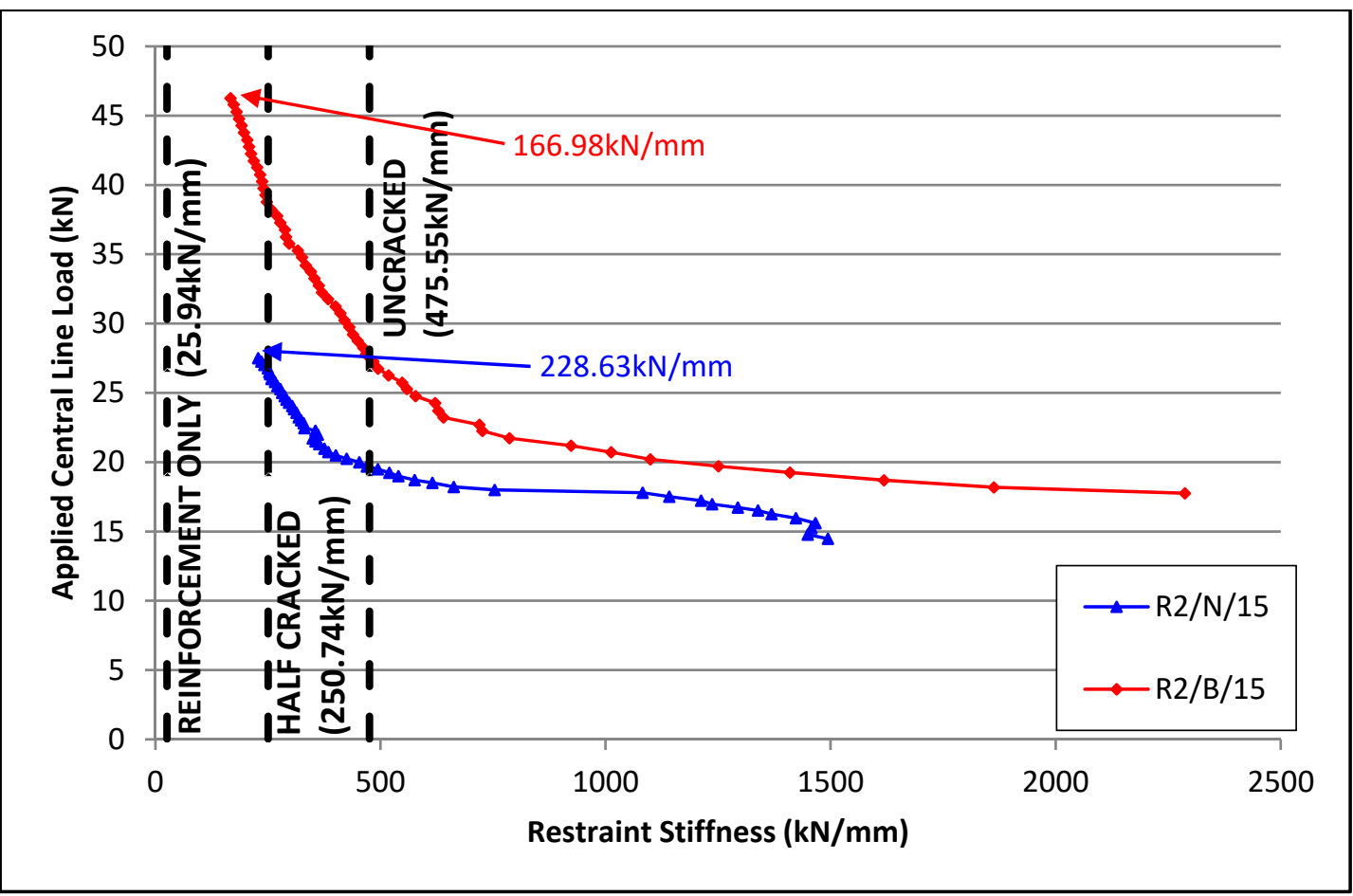

Figure 21: Variation of equivalent restraint stiffness with applied load for deep slabs with high restraint 


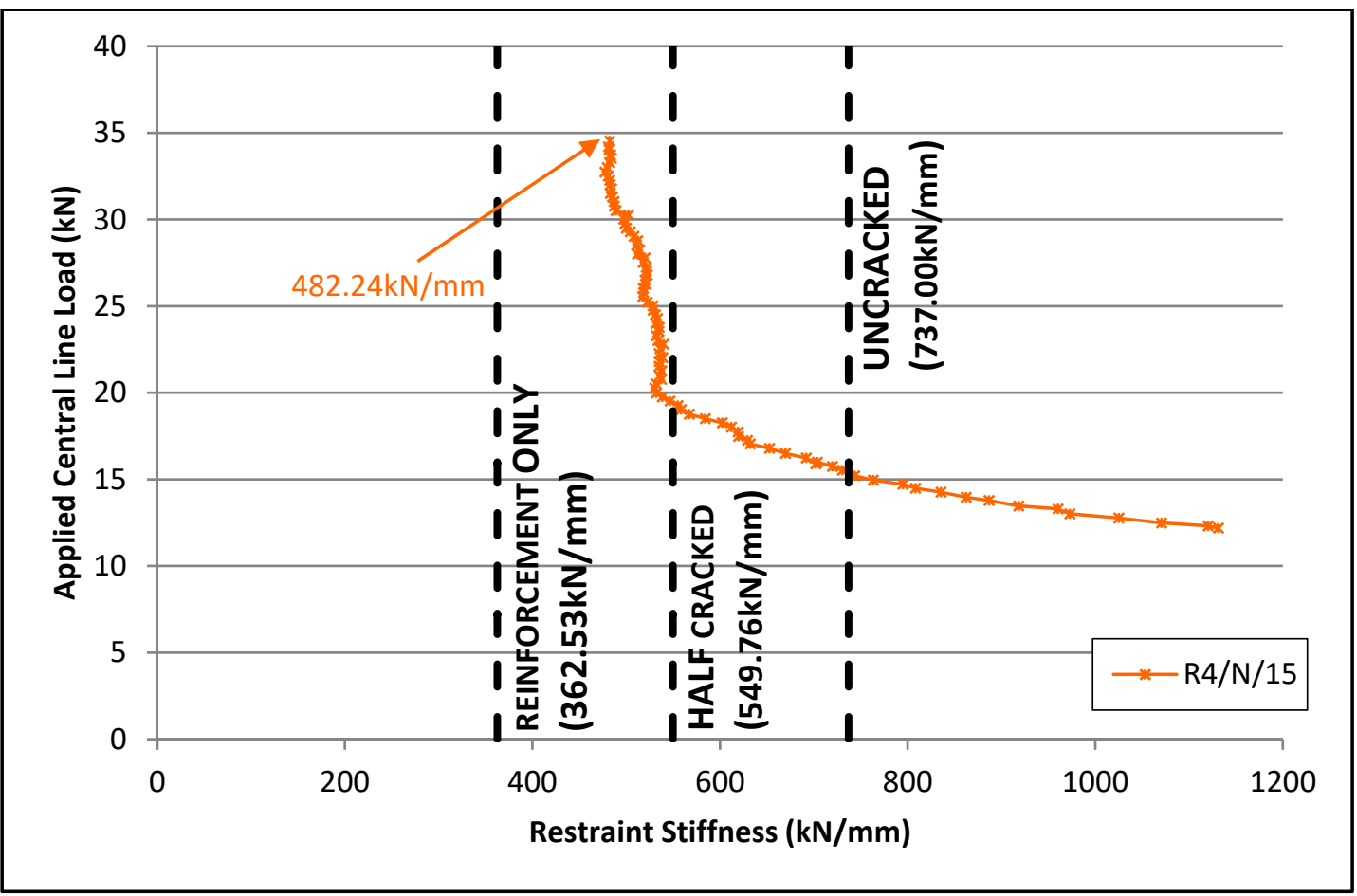

Figure 22: Variation of equivalent restraint stiffness with applied load for deep slab with very high restraint

Experimental failure load versus restraint stiffness trends were compared for all slabs over a range of restraint levels. These show, in the following subsections, how experimental results compared with initial axial estimates.

\subsection{Comparison of methods to determine restraint stiffness}

Restraint stiffness versus experimental failure load trends were plotted for all slabs with a particular emphasis on comparing preliminary stiffness estimates with experimentally derived values. These are shown in Figures $23-25$ and $26-27$ for shallow slabs and deeper slabs respectively. They show that failure capacity varied approximately linearly with restraint stiffness in all cases irrespective of span-to-depth ratio.

In unstrengthened restrained slabs, experimental stiffnesses were generally slightly lower than the preliminary 'uncracked' condition across all restraint levels, as shown in Figure 23. This was also the case in shallow BFRP strengthened restrained slabs, except for those with low (i.e. 'R05') restraint, as shown in Figure 24. In CFRP strengthened shallow restrained slabs, experimental stiffness trends were near identical to preliminary 'half cracked' estimates, as shown in Figure 25. Given the performance of shallow FRP strengthened restrained slabs, the results therefore 
showed that an initial assumption of purely axial 'half cracked' restraint can lead to reasonable estimates of slab capacity, with an increased level of safety associated with using BFRP strengthening.

Investigations into unstrengthened deep restrained slabs, as illustrated in Figure 26, showed that experimental stiffnesses were slightly lower than preliminary 'half cracked' estimates. A similar comparison was observed in deep BFRP strengthened restrained slabs, as illustrated in Figure 27. Hence, the results showed that restraint stiffnesses approximately two thirds between 'reinforcement only' and 'half cracked' conditions produced reasonable estimates of actual stiffness at peak capacity.

A comparison of experimental stiffness and failure values with strengthening material is illustrated in Figure 28. This shows that capacity increased approximately linearly with restraint stiffness, and with similar gradients depending upon span-to-depth ratio, for each strengthening material. However, small drops in capacity between simply supported and low restraint cases for strengthened slabs are evident. This may be attributed to slightly lower concrete strength and insufficient concrete compaction in the case of $R 05 / B / 20$. However, based upon concrete strength alone, the flexural component of $\mathrm{R} 05 / \mathrm{C} / 20$ was predicted to be higher than the overall experimental capacity of $15.77 \mathrm{kN}$. This suggests that low levels of in-plane restraint in shallow strengthened slabs may also an inhibitive effect on the full development of flexural capability.

Strengthening shallow restrained slabs with BFRP was more effective in increasing capacity compared with CFRP in these investigations due to the higher strength of BFRP bars used in this research, as detailed in Table 3. However, whilst a higher gradient for " $1 / \mathrm{d}=20$, CFRP" compared with " $/ \mathrm{d}=20$, BFRP" in Figure 28 would suggest that CFRP is the more effective strengthening material, the intersection point between these two lines (i.e. $k_{r}=308 \mathrm{kN} / \mathrm{mm}$ ) indicates that this can only occur in the presence of restraint stiffness levels far in excess of what can reasonably be anticipated within existing building structures. Hence BFRP strengthening is considered to provide larger increases in capacity in restrained slabs in all practical situations.

In strengthening deep restrained slabs with BFRP, the step in capacity between unstrengthened and strengthened states was significantly higher than that in shallow slabs. For example, as shown in Figure 28 , at $k_{r}=100 \mathrm{kN} / \mathrm{mm}$, the capacity step due to BFRP strengthening in shallow slabs was $11.9 \mathrm{kN}(137 \%$, i.e. $8.7 \mathrm{kN}$ to $20.6 \mathrm{kN})$, while in deep slabs this increased to $24.6 \mathrm{kN}(133 \%$, i.e. $18.5 \mathrm{kN}$ to $43.1 \mathrm{kN})$. As the gradients identified in each experimental case were similar, depending upon slab 
depth, this indicated that FRP strengthening and CMA effects due to restraint were generally cumulative, but with some overlap.

In shallow slabs BFRP and CFRP strengthening provided capacity increases of approximately $11.9 \mathrm{kN}(136 \%)$ and $9.3 \mathrm{kN}(107 \%)$ respectively at $100 \mathrm{kN} / \mathrm{mm}$ in Figure 28 compared to the unstrengthened sample's $8.7 \mathrm{kN}$ capacity at $100 \mathrm{kN} / \mathrm{mm}$, whilst each $100 \mathrm{kN} / \mathrm{mm}$ of additional restraint in strengthened slabs provided an increase in failure capacity of between $0.8 \mathrm{kN}(3.8 \%)$ with BFRP and $2 \mathrm{kN}(11.3 \%)$ with CFRP. This indicated that FRP strengthening was significantly more effective in increasing strength than CMA effects and that BFRP strengthening had a more direct effect on increasing flexural capacity than CFRP, but that CFRP had a more significant effect on increasing restraint stiffness than BFRP, causing greater subsequent increases in arching effects leading to higher capacity.

Both shallow and deep slabs were shown to achieve capacity increases due to both CMA effects and FRP strengthening. However, as the slope of experimental restraint stiffness versus experimental failure load trends were significantly higher (i.e. over $200 \%$ higher) with deep slabs, compared with shallow slabs, this indicated that deep slabs were significantly more efficient in increasing their capacity due to arching effects. However, even in unstrengthened cases for shallow slabs with 'regular' levels of restraint, a $48 \%$ increase in capacity was observed between slabs $\mathrm{S} / \mathrm{N} / 20(7.02 \mathrm{kN})$ and $\mathrm{R} 1 / \mathrm{N} / 20(10.43 \mathrm{kN})$. Therefore, as this represents a significant level of additional strength in real building structure slabs, they should not be ignored. 


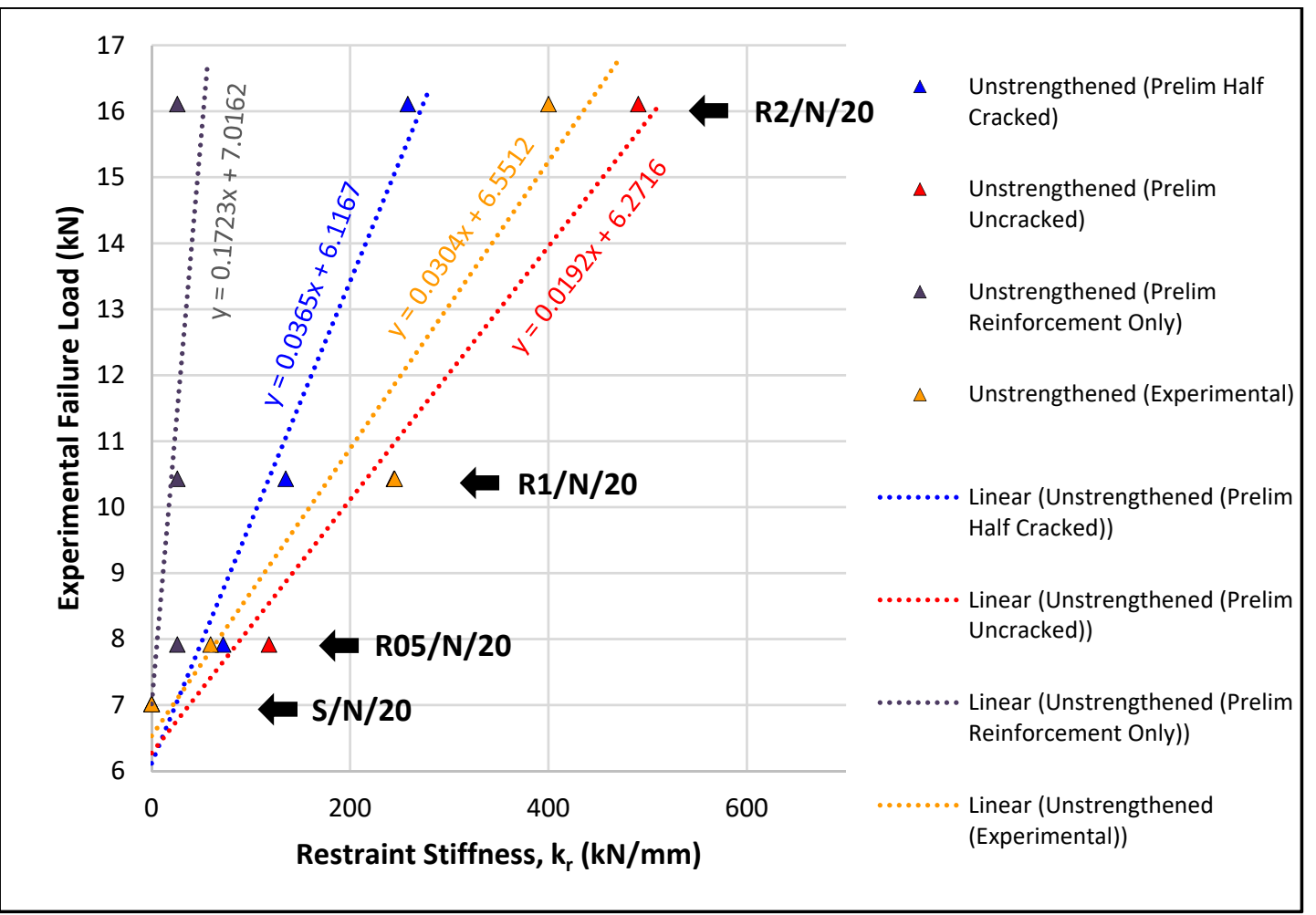

Figure 23: Variation of restraint stiffnesses with experimental failure load for shallow unstrengthened restrained slabs

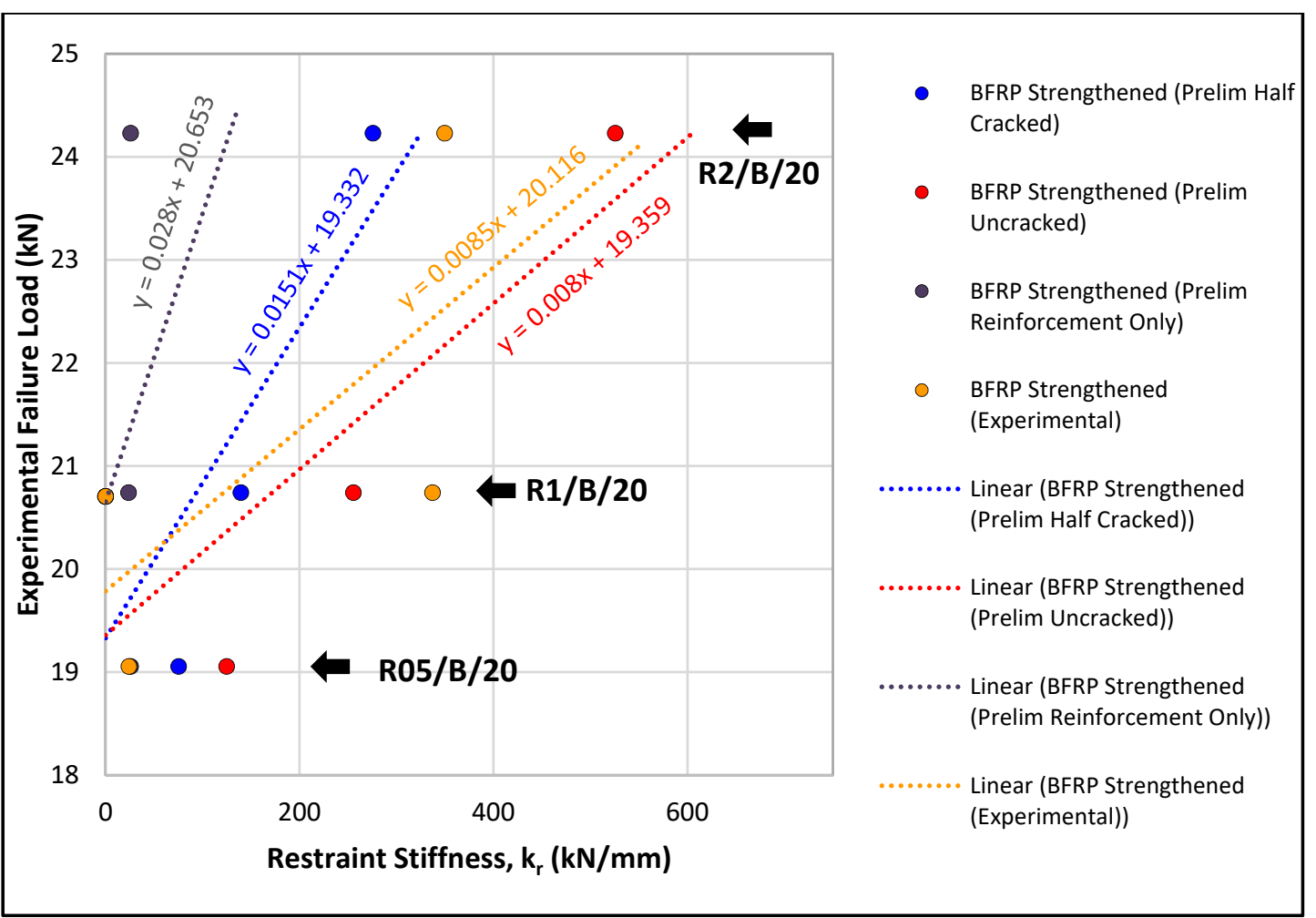

Figure 24: Variation of restraint stiffnesses with experimental failure load for shallow BFRP strengthened restrained slabs 


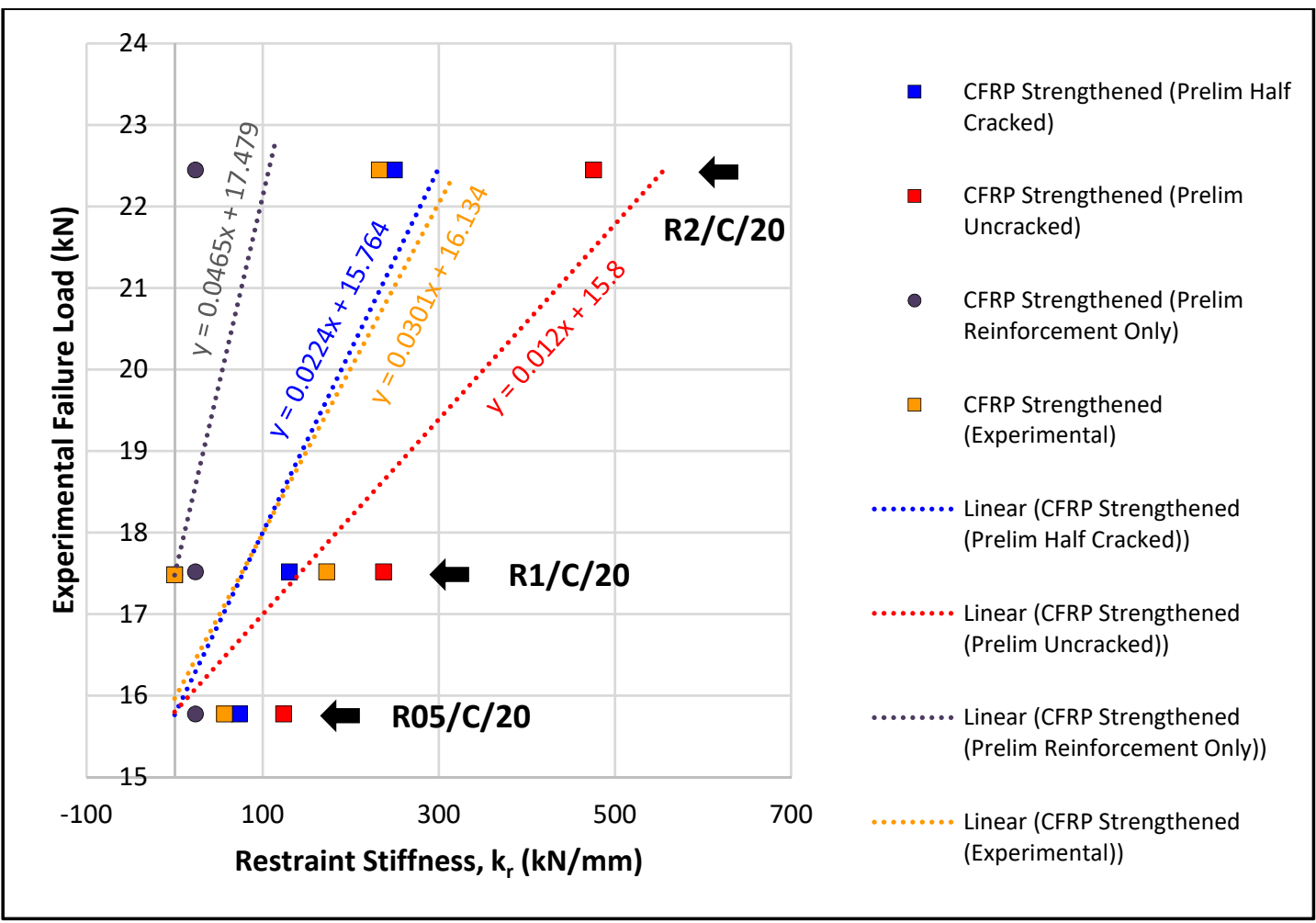

Figure 25: Variation of restraint stiffnesses with experimental failure load for shallow CFRP strengthened restrained slabs

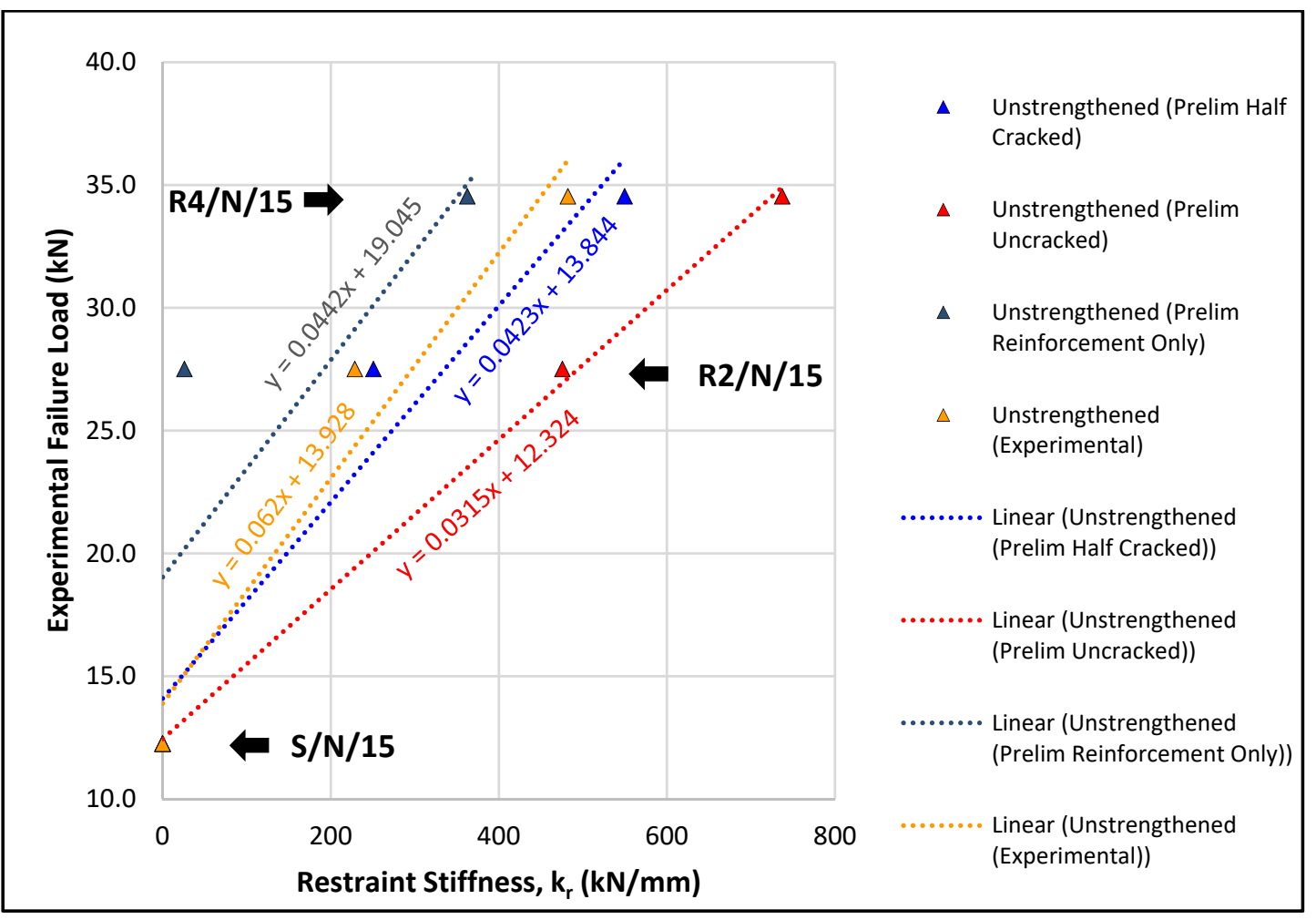

Figure 26: Variation of restraint stiffnesses with experimental failure load for deep unstrengthened restrained slabs 


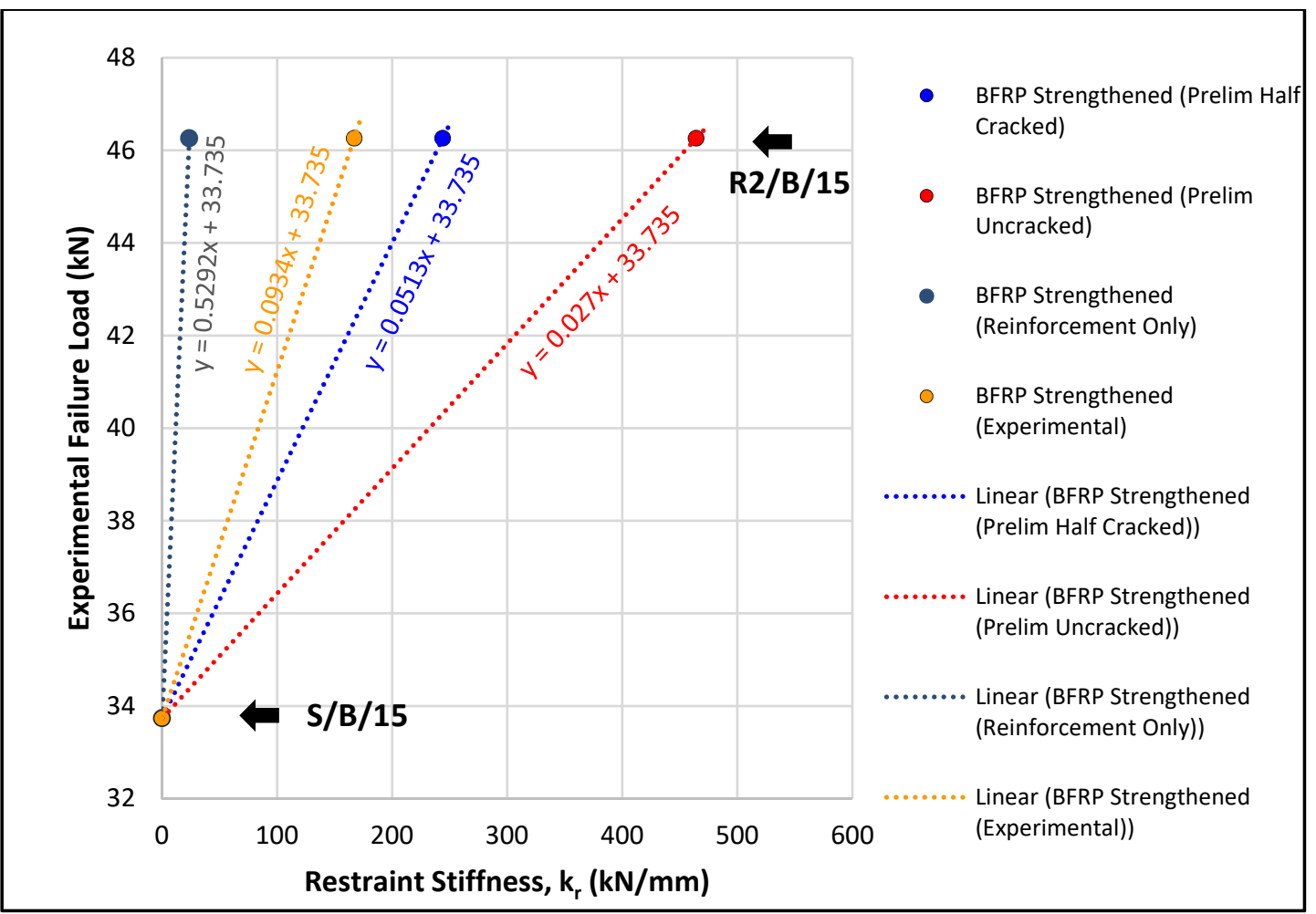

Figure 27: Variation of restraint stiffnesses with experimental failure load for deep BFRP strengthened restrained slabs

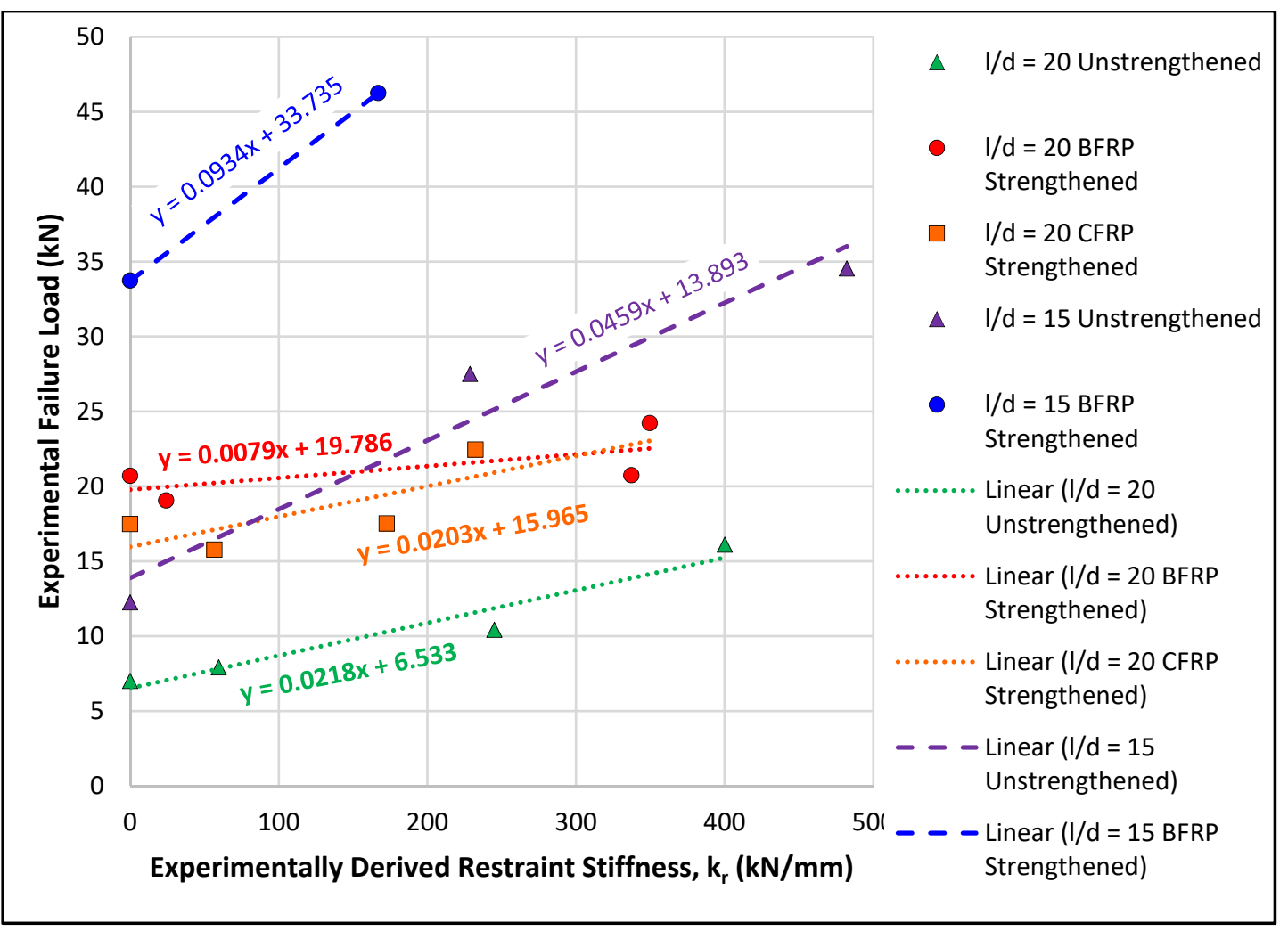

Figure 28: Variation of experimental restraint stiffness with experimental failure load for restrained slabs 
Experimental slab capacities are presented in Table 9 alongside American [60] [61] and European [62] [63] code predictions and the results showed that both FRPs were highly effective strengthening materials, although BFRP had a greater effect in this regard, compared with CFRP, due to its higher strength in these investigations.

By considering experimental stiffnesses in conjunction with preliminary axial estimates of 'uncracked' and 'half cracked' conditions, the following estimates of actual stiffness can be made:

i. Unstrengthened and BFRP strengthened shallow slabs:

$$
\boldsymbol{k}_{r, \text { Actual }}=\frac{\left\{\boldsymbol{m}_{U C} \boldsymbol{k}_{U C}+C_{U C}-C_{E X}\right\}}{m_{E X}}
$$

ii. Shallow CFRP strengthened restrained slabs:

$$
\boldsymbol{k}_{r, \text { Actual }}=\frac{\left\{\boldsymbol{m}_{H C} \boldsymbol{k}_{r, H C}+C_{H C}-C_{E X}\right\}}{m_{E X}}
$$

iii. Deep slabs, both unstrengthened and strengthened with BFRP:

$$
\boldsymbol{k}_{r, \text { Actual }}=\frac{\left\{\boldsymbol{m}_{H C} \boldsymbol{k}_{r, H C}+C_{H C}-C_{E X}\right\}}{m_{E X}}
$$

$$
\begin{aligned}
& \text { where: } \quad \begin{array}{l}
k_{r, A c t u a l}=\text { Actual restraint stiffness }(\mathrm{kN} / \mathrm{mm}) \\
k_{r, H C}=\text { Estimate of half cracked restraint stiffness }(\mathrm{kN} / \mathrm{mm}) \\
k_{r, U C}=\text { Estimate of uncracked restraint stiffness }(\mathrm{kN} / \mathrm{mm}) \\
m_{H C}=\text { Gradient of half cracked linear trend } \\
m_{U C}=\text { Gradient of uncracked linear trend } \\
m_{E X}=\text { Gradient of experimental linear trend } \\
c_{H C}=\text { Vertical intersect of half cracked linear trend } \\
c_{U C}=\text { Vertical intersect of uncracked linear trend } \\
c_{E X}=\text { Vertical intersect of experimental linear trend }
\end{array}
\end{aligned}
$$

The values outlined in Table 8, established from an analysis of the results obtained in these investigations, may be used by designers in applying equations (35-37) along with their estimates for half cracked and uncracked restraint stiffness. 
It is notable that the capacity of restrained strengthened slabs were initially overestimated by up to $34 \%$ in strengthened restrained slabs, as shown in Table 9 , when applying the 'pure' arching theory using experimentally derived restraint stiffness values. This suggested that the beneficial effects of FRP strengthening and arching tended to overlap to some extent. A review of the data indicated that a reduction of $25 \%$ to the efficiency of FRP strengthening in restrained slabs, along with evaluating restraint stiffness in accordance with the recommendations in equations (35 - 37) and Table 8 led to more accurate capacity (i.e. 'design arching') predictions, as outlined in Table 9.

Table 8: Recommended constant values for use in estimating restraint stiffness in design scenarios

\begin{tabular}{|c|c|}
\hline \multicolumn{2}{|c|}{ Span-to-depth $=20$, Unstrengthened } \\
\hline$m_{U C}$ & 0.0192 \\
\hline$m_{E X}$ & 0.0304 \\
\hline$c_{U C}$ & $6.2716 \mathrm{kN}$ \\
\hline$c_{E X}$ & $6.5512 \mathrm{kN}$ \\
\hline \multicolumn{2}{|c|}{ Span-to-depth $=20$, BFRP Strengthened } \\
\hline$m_{U C}$ & 0.0085 \\
\hline$m_{E X}$ & 0.0080 \\
\hline$c_{U C}$ & $19.359 \mathrm{kN}$ \\
\hline$c_{E X}$ & $20.116 \mathrm{kN}$ \\
\hline \multicolumn{2}{|c|}{ Span-to-depth $=20$, CFRP Strengthened } \\
\hline$m_{H C}$ & 0.0224 \\
\hline$m_{E X}$ & 0.0301 \\
\hline$c_{H C}$ & $15.764 \mathrm{kN}$ \\
\hline$c_{E X}$ & $16.134 \mathrm{kN}$ \\
\hline \multicolumn{2}{|c|}{ Span-to-depth $=15$, Unstrengthened } \\
\hline$m_{H C}$ & 0.0423 \\
\hline$m_{E X}$ & 0.0620 \\
\hline$c_{H C}$ & $13.844 \mathrm{kN}$ \\
\hline$c_{E X}$ & $13.928 \mathrm{kN}$ \\
\hline \multicolumn{2}{|c|}{ Span-to-depth $=15$, BFRP Strengthened } \\
\hline$m_{H C}$ & 0.0513 \\
\hline$m_{E X}$ & 0.0934 \\
\hline$c_{H C}$ & $33.735 \mathrm{kN}$ \\
\hline$c_{E X}$ & $33.735 \mathrm{kN}$ \\
\hline
\end{tabular}

Hence, for the successful application of this theory within an engineering design context, the following guidelines are recommended: 
1. In all cases where in-plane restraint is present, equations (35-37) should be employed along with recommended constant values given in Table 8 to establish restraint $k_{r}$.

2. For simply supported slabs: Full efficiency of FRP should be adopted.

3. Where FRP strengthening is applied where in-plane restraint is also considered, an efficiency factor of 0.75 should be applied to FRP.

This approach compares favourably with European [62] [63] and American [60] [61] 'flexural only' estimates and illustrates the beneficial effects of including CMA effects in estimating slab capacity using the QUB Arching Theory.

Slab R2/C/20 shall now be evaluated in accordance with these recommendations and with the following material properties:

- Yield strength of steel reinforcement, $f_{\mathrm{yk}}=549.30 \mathrm{~N} / \mathrm{mm}^{2}$

- Young's Modulus for Steel Reinforcement, $E_{s}=201.94 \mathrm{kN} / \mathrm{mm}^{2}$

- Compressive strength of concrete, $\mathrm{f}_{\mathrm{ck}, \mathrm{cube}}=43.12 \mathrm{~N} / \mathrm{mm}^{2}$

$$
\begin{aligned}
& k_{H C}=2 \times k_{\text {Beam }}=2 \times\left\{\frac{E_{c, \text { Beam }} A_{C, \text { Beam }}}{L_{\text {Beam }}}+\frac{E_{S, \text { Beam }} A_{S, \text { Beam }}}{L_{\text {Beam }}}\right\} \quad \text { from equation (25) } \\
& k_{H C}=2 \times k_{\text {Beam }}=2 \times\left\{\frac{E_{c, \text { Beam }} A_{c, \text { Beam }}}{L_{\text {Beam }}}+\frac{E_{S, \text { Beam }} A_{S, \text { Beam }}}{L_{\text {Beam }}}\right\} \\
& k_{H C}=2 \times\left\{\frac{0.5 \times(4230 \times \sqrt{43.12}) \times\left(100 \times 147-4 \times\left(\frac{\pi \times 6^{2}}{4}\right)\right)}{1792}+\frac{210940 \times\left(\frac{\pi \times 6^{2}}{4}\right) \times 4}{1792}\right\} \\
& k_{H C}=252728 \mathrm{~N} / \mathrm{mm} \approx 253 \mathrm{kN} / \mathrm{mm}
\end{aligned}
$$

Hence, from equation (36):

$$
\begin{aligned}
& k_{r, \text { Design }}=\frac{\left\{m_{H C} k_{H C}+C_{H C}-C_{E X}\right\}}{m_{E X}}=\frac{\{0.0224 \times 253+15.764-16.134\}}{0.0301} \\
& \boldsymbol{k}_{\boldsymbol{r}, \text { Design }}=\mathbf{1 7 6} \boldsymbol{k N} / \mathbf{m m}
\end{aligned}
$$

Calculations to establish the simply supported slab capacity for slab R2/C/20 based on Eurocode and $\mathrm{ACl}$ codes are not given here, as they can be easily determined by following these codes. However, these show that their mid-span point load capacity is $16.64 \mathrm{kN}$ and $16.46 \mathrm{kN}$ by $\mathrm{EC} 2$ and $\mathrm{ACl}$ respectively. This shall now be compared with the determination of the slab capacity using arching theory incorporating the 
estimated restraint of $176 \mathrm{kN} / \mathrm{mm}$ found above along with a CFRP efficiency factor of 0.75 .

\section{Step 1}

\section{Stiffness parameters}

Restraint stiffness $\quad k_{r}=176 \mathrm{kN} / \mathrm{mm}$

\section{Bending capacity}

Proportional depth of stress block factor:

$\beta=1-0.003 f_{\text {ck,cube }}$ but $\leq 0.9$

$\beta=1-0.003 \times 43.12=0.871$

Depth of neutral axis: $x=\frac{A_{S} f_{y k}+\xi_{F R P} A_{F R P} f_{F R P, k}}{0.67 f_{c k, c u b e} \beta b}=\frac{84.82 \times 549.30+0.75 \times 58.95 \times 990.42}{0.67 \times 43.12 \times 0.871 \times 680}=5.28 \mathrm{~mm}$ Moment capacity due to bending:

$M_{b}=\left(A_{s} f_{y k}\right)\left[d-\frac{\beta x}{2}\right]+\left(\xi_{F R P} A_{F R P} f_{F R P, k}\right)\left[d_{F R P}-\frac{\beta x}{2}\right]$

Where $\xi_{F R P}=$ FRP efficiency factor

$M_{b}=(84.82 \times 549.30)\left[65.30-\frac{0.871 \times 5.28}{2}\right]+(0.75 \times 58.95 \times 990.42)\left[79.48-\frac{0.871 \times 5.28}{2}\right]$

$M_{b}=6.31 \mathrm{kNm}$

Load corresponding to bending moment capacity:

$P_{b}=\frac{4 M_{b}}{L}=\frac{4 \times 6.31}{1.792}=14.10 k N$

\section{Step 2}

\section{Arching section}

Depth available for arching: $d_{1}=\frac{h-2 x \beta}{2}=\frac{83.3-2 \times 5.28 \times 0.871}{2}=37.05 \mathrm{~mm}$

\section{Affine strip}

Area of concrete due to arching (assume $\alpha=1$ for first iteration):

$A=\alpha b d_{1}=1 \times 680 \times 37.05=25195 \mathrm{~mm}^{2}$ 
Equivalent rigid half arch span:

$$
L_{r}=L_{e}\left[\frac{E_{c} A}{k_{r} L_{e}}+1\right]^{1 / 3}=\left(\frac{1792}{2}\right) \times\left[\frac{4230 \times \sqrt{43.12} \times 25195}{\left(176 \times 10^{3}\right) \times\left(\frac{1792}{2}\right)}+1\right]^{1 / 3}=1472.42 \mathrm{~mm}
$$

\section{Arching parameters}

Ultimate compressive strain in concrete:

$\varepsilon_{u}=0.0043-\left[f_{c k, c u b e}-60\right] \times\left(2.5 \times 10^{-5}\right) \quad$ but $\leq 0.0043$

$\varepsilon_{u}=0.0043-[43.12-60] \times\left(2.5 \times 10^{-5}\right)=0.0047>0.0043$

Therefore let $\varepsilon_{u}=0.0043$

Concrete plastic strain:

$\varepsilon_{c}=2 \varepsilon_{u}(1-\beta)=2 \times 0.0043 \times(1-0.871)=0.0011$

McDowell's non-dimensional geometry and material factor:

$R=\frac{\varepsilon_{c} L_{r}^{2}}{4 d_{1}^{2}}=\frac{0.0011 \times 1472.42^{2}}{4 \times 37.05^{2}}=0.434$

\section{Deformation}

$R>0.26 \quad$ Therefore: $\quad u=0.31$

\section{Contact depth}

$\alpha=1-\frac{u}{2}=1-\frac{0.31}{2}=0.845$

Therefore, the refined area of concrete in arching becomes:

$A=\alpha b d_{1}=0.845 \times 680 \times 37.05=21288.90 \mathrm{~mm}^{2}$

Iterations within step 2 results in the following values:

$u=0.31$

$\varepsilon_{u}=0.00430$

$\alpha=0.845$

$\varepsilon_{c}=0.00111$

$A=21288.39 \mathrm{~mm}^{2}$

$R=0.463$

$L_{r}=1511.64 \mathrm{~mm}$ 


\section{Step 3}

\section{Arching capacity}

$R>0.26 \quad$ Therefore, moment ratio:

$M_{r}=\frac{0.3615}{R}=\frac{0.3615}{0.463}=0.7808$

Equivalent rigid arching moment of resistance:

$M_{a r}=0.168 b f_{c k, c u b e} d_{1}^{2} M_{r}\left(\frac{L_{e}}{L_{r}}\right)$

$M_{a r}=0.168 \times 680 \times 43.12 \times 37.05^{2} \times 0.7808 \times\left(\frac{(1792 / 2)}{1511.64}\right)=3.129 \mathrm{kNm}$

Elastic arching moment of resistance:

$M_{a}=M_{a r}\left(\frac{L_{e}}{L_{r}}\right)=3.129 \times\left(\frac{(1792 / 2)}{1511.64}\right)=1.855 \mathrm{kNm}$

Load corresponding to arching capacity:

$P_{a}=\frac{4 M_{a}}{L}=\frac{4 \times 1.855}{1.792}=4.14 k N$

\section{Ultimate capacity}

$P_{p}=P_{a}+P_{b}=4.14+14.10=18.24 k N$

Thus, by considering the in-plane restraint inherent within this slab, and the contribution due to FRP strengthening, a capacity increase of approximately $10.2 \%$ was established in comparison with Eurocode and $\mathrm{ACl}$ predictions. An excel spreadsheet was used to automate the iterative calculations above. Generally, only a few iterations were required to obtain stable estimations, but the Microsoft Excel macro was set to allow up to 100 iterations. 
Table 9: Comparison of predicted capacities and experimental failure loads for test slabs

\begin{tabular}{|c|c|c|c|c|c|c|c|c|c|}
\hline Slab Code & $\begin{array}{l}\text { EC2 Predicted } \\
\text { Load, } P_{\mathrm{p}, \mathrm{EC} 2} \\
(\mathrm{kN})\end{array}$ & $\begin{array}{l}\text { ACI Predicted } \\
\text { Load, } \mathrm{P}_{\mathrm{p}, \mathrm{ACl}} \\
(\mathrm{kN})\end{array}$ & $\begin{array}{l}\text { Predicted Load Using Arching } \\
\text { Theory Incorporating } \\
\text { Experimental Restraint Stiffness, } \\
\mathbf{P}_{\mathrm{p}, \text { pure arching }}(\mathrm{kN})\end{array}$ & $\begin{array}{c}\text { Predicted Load Using Arching } \\
\text { Theory Incorporating Design } \\
\text { Estimated Restraint Stiffness, } \\
\mathbf{P}_{\mathrm{p}, \text { design arching }}(\mathrm{kN})\end{array}$ & $\begin{array}{l}\text { Experimental } \\
\text { Failure Load, } \mathrm{P}_{\mathrm{t}} \\
\quad(\mathrm{kN})\end{array}$ & $\begin{array}{l}P_{p} / P_{t} \\
(E C 2)\end{array}$ & $\begin{array}{l}\mathrm{P}_{\mathrm{p}} / \mathrm{P}_{\mathrm{t}} \\
(\mathrm{ACl})\end{array}$ & $\begin{array}{c}\mathbf{P}_{\mathrm{p}} / \mathbf{P}_{\mathrm{t}} \\
\text { (Pure } \\
\text { Arching) }\end{array}$ & $\begin{array}{c}\mathbf{P}_{\mathrm{p}} / \mathbf{P}_{\mathbf{t}} \\
\text { (Design } \\
\text { Arching) }\end{array}$ \\
\hline$S / N / 20$ & 6.09 & 6.08 & 6.07 & 6.07 & 7.02 & 0.87 & 0.87 & 0.87 & 0.87 \\
\hline S/N/15 & 11.61 & 11.60 & 11.58 & 11.58 & 12.25 & 0.95 & 0.95 & 0.95 & 0.95 \\
\hline$S / C / 20$ & 16.65 & 16.47 & 16.54 & 16.54 & 17.48 & 0.95 & 0.94 & 0.95 & 0.95 \\
\hline $\mathrm{S} / \mathrm{B} / 20$ & 20.77 & 20.69 & 20.64 & 20.64 & 20.70 & 1.00 & 1.00 & 1.00 & 1.00 \\
\hline S/B/15 & 37.61 & 37.51 & 37.33 & 37.33 & 33.73 & 1.11 & 1.11 & 1.11 & 1.11 \\
\hline R1/N/20 & 6.09 & 6.08 & 13.09 & 10.11 & 10.43 & 0.58 & 0.58 & 1.26 & 0.97 \\
\hline $\mathrm{R} 0.5 / \mathrm{N} / 20$ & 6.09 & 6.09 & 7.48 & 7.65 & 7.92 & 0.77 & 0.77 & 0.94 & 0.97 \\
\hline $\mathrm{R} 2 / \mathrm{N} / 20$ & 6.09 & 6.08 & 17.11 & 14.61 & 16.11 & 0.38 & 0.38 & 1.06 & 0.91 \\
\hline $\mathrm{R} 2 / \mathrm{N} / 15$ & 11.59 & 11.57 & 26.85 & 22.59 & 27.51 & 0.42 & 0.42 & 0.98 & 0.82 \\
\hline R4/N/15 & 12.71 & 12.69 & 39.84 & 35.46 & 34.54 & 0.37 & 0.37 & 1.15 & 1.03 \\
\hline $\mathrm{R} 1 / \mathrm{C} / 20$ & 16.64 & 16.46 & 20.40 & 15.95 & 17.52 & 0.95 & 0.94 & 1.16 & 0.91 \\
\hline $\mathrm{R} 1 / \mathrm{B} / 20$ & 21.25 & 21.19 & 27.81 & 21.38 & 20.74 & 1.02 & 1.02 & 1.34 & 1.03 \\
\hline $\mathrm{R} 0.5 / \mathrm{C} / 20$ & 16.74 & 16.52 & 17.67 & 14.93 & 15.77 & 1.06 & 1.05 & 1.12 & 0.95 \\
\hline $\mathrm{R} 0.5 / \mathrm{B} / 20$ & 20.69 & 20.63 & 20.87 & 17.61 & 19.05 & 1.09 & 1.08 & 1.10 & 0.92 \\
\hline $\mathrm{R} 2 / \mathrm{C} / 20$ & 16.64 & 16.46 & 21.77 & 18.24 & 22.45 & 0.74 & 0.73 & 0.97 & 0.81 \\
\hline $\mathrm{R} 2 / \mathrm{B} / 20$ & 20.69 & 20.63 & 27.60 & 26.68 & 24.23 & 0.85 & 0.85 & 1.14 & 1.10 \\
\hline \multirow[t]{4}{*}{$\mathrm{R} 2 / \mathrm{B} / 15$} & 38.63 & 37.56 & 46.11 & 38.66 & 46.26 & 0.84 & 0.81 & 1.00 & 0.84 \\
\hline & & & & & Average $=$ & 0.82 & 0.82 & 1.06 & 0.95 \\
\hline & & & & & andard Deviation = & 0.25 & 0.24 & 0.13 & 0.09 \\
\hline & & & & & cient of variation $=$ & 0.06 & 0.06 & 0.01 & 0.01 \\
\hline
\end{tabular}




\section{Discussion and conclusions}

All restraint beams cracked during slab loading due to combined axial and bending effects which resulted in altering in-plane slab restraint as loading increased. These restraint stiffnesses reduced rapidly under increased loading and were compared with simplified axial estimates based on restraint beam cross sectional geometries.

The research has shown that significant additional capacity can be either 'found' within existing reinforced concrete floor slabs by accounting for the restraining effects of their adjacent parallel floor beams or, if necessary, can be further increased by the addition of low proportions; approximately $0.10 \%$, of CFRP/BFRP strengthening applied using the near surface mounted installation technique.

Results showed that even the lowest geometric estimate of restraining stiffness (based upon only steel reinforcement acting in tension, with no contribution from concrete) produced capacity estimates which were generally lower than experimentally derived values. Hence, it can be concluded that capacity predictions obtained using the very lowest 'reinforcement only' restraint beam axial estimate can still lead to safe estimates of restrained slab capacity; exceeding predictions based on common codes for all of the slabs considered in the research thus, providing some additional capacity not included in current codes.

Finally, the investigations demonstrated that using unmodified experimental restraint stiffness values along with the QUB arching theory resulted in slab capacity predictions which were generally quite good, but with some values which were beyond an acceptably safe limit. Furthermore, experimental results showed that arching and FRP strengthening were largely separate phenomena, but with an overlapping cumulative effect on increasing slab capacity. Therefore, recommendations were developed for practicing engineers to apply FRP strengthening and arching behaviour within a design context in order to establish closer safe estimates of slab capacity compared with existing design codes.

\section{Acknowledgements}

The authors wish to express their sincere appreciation to MagmaTech for the supply of all basalt and carbon fibre bars used in the research and to The Northern Ireland Department for Employment and Learning (DEL) without which the research could not have been carried out. 


\section{References}

[1] M. Kelly, Britain's building stock - a carbon challenge (Presentation, 2008)

[2] UNEP, 2016 UNEP Sustainable buildings and climate initiative: Why buildings. United Nations environment programme (UNEP) (2016) Accessed on January 2016 at http://www.unep.org/sbci/AboutSBCl/Background.asp

[3] Conejos, S., Langston, C., Smith J (2015) Enhancing sustainability through designing for adaptive reuse from the outset: A comparison of adaptSTAR and Adaptive Reuse Potential (ARP) models Facilities, 33 (9/10) (2015), pp. 531-552

[4] Wilkinson, S. J. and Remoy, H., (2017) Adaptive reuse of Sydney offices and sustainability. Sustainable Buildings, 2, 6.

[5] Assefa, G. and Ambler, C. (2016) To demolish or not to demolish: Life cycle consideration of repurposing buildings. Sustainable Cities and Society, Volume 28, January 2017, Pages 146-153.

[6] Yeheyis, M., Hewage, K., Alam, M.S., Eskicioglu, C., Sadiq R. (2013) An overview of construction and demolition waste management in Canada: A lifecycle analysis approach to sustainability Clean Technologies and Environmental Policy, 15 (1) (2013), pp. 81-91

[7] de Waal, L., Fernando, D., Van Thuan, N., Cork, R., Foote, J. (2017) FRP strengthening of 60 year old pre-stressed concrete bridge deck units. Engineering Structures, Volume 143, 15 July 2017, Pages 346-357.

[8] Darby, A, Ibell, T, Evernden, M, Tajaddini, A \& Raicic, V. (2017) 'Challenges for strengthening concrete structures in flexure, shear and compression using fibre reinforced polymers' Paper presented at International Conference on Structural Engineering and Construction Management, 2017, Kandy, Sri Lanka, 8/12/17 10/01/18, .

[9] Foster, R, Brindley, M, Lees, J, Ibell, T, Morley, C, Darby, A \& Evernden, M. (2017) 'Experimental investigation of reinforced concrete T-beams strengthened in shear with externally bonded CFRP sheets' ASCE Journal of Composites for Construction, vol. 21, no. 2, 04016086. DOI: 10.1061/(ASCE)CC.1943-5614.0000743 
[10] Foster, Robert M. (2015) Shear Strengthening of Reinforced Concrete Tbeams with Carbon Fibre Reinforced Polymer Fabrics. PhD Thesis, Department of Engineering, University of Cambridge, University of Cambridge.

[11] Moon, J., Mahmoud, M., Taha, R. and Kim, J. J. (2017) Flexural Strengthening of RC Slabs Using a Hybrid FRP-UHPC System Including Shear Connector, Advances in Materials Science and Engineering, vol. 2017, Article ID 4387545, 7 pages.

[12] Gopu, V. and Ziehl, P. H., (2016) Strengthening of Bridge Beams using Fiber Reinforced Polymers (FRP). 1st ed. Louisiana, USA: Louisiana Department of Transportation and Development Louisiana Transportation Research Center.

[13] Sheikh, S. A. (2001) Performance of concrete structures retrofitted with fibre reinforced polymers. Engineering Structures, Volume 24, Issue 7, Pages 869-879.

[14] Teng, J.G., Chen, J. F., Smith, S. T., Lam, L., Chen, J-F. (2001) FRP: Strengthened RC Structures. 1st Ed. England: John Wiley \& Sons, Ltd.

[15] Aidoo J., Harries K.A., Petrou M.F. (2006) Full-Scale Experimental Investigation of Repair of Reinforced Concrete Interstate Bridge Using CFRP Materials. Journal of Bridge Engineering Vol. 11 (No. 3, May 2006) p350

[16] Hawileh, R. A., Rasheed, H. A., Abdalla, J. A., Al-Tamimi, A. K. (2014) Behavior of reinforced concrete beams strengthened with externally bonded hybrid fiber reinforced polymer systems. Materials \& Design, Volume 53, January 2014, Pages 972-982.

[17] Wu, Y., Huang, Y. (2008) Hybrid Bonding of FRP to Reinforced Concrete Structures. Journal of Composites for Construction, Volume 12, Issue 3, pp 266-273.

[18] Sharaky, I. A., Torres, L., Comas, J., Barris, C. (2014) Flexural response of reinforced concrete (RC) beams strengthened with near surface mounted (NSM) fibre reinforced polymer (FRP) bars. Composite Structures, Volume 109, March, Pages 822.

[19] Parvin, A.; Syed Shah, T. (2016) Fiber Reinforced Polymer Strengthening of Structures by Near-Surface Mounting Method. Polymers, 8, 298. 
[20] Loring, H. B., Davids, W. G., (2015) Mechanically fastened hybrid composite strips for flexural strengthening of concrete beams. Construction and Building Materials, 76, pp118-129.

[21] De Lorenzis L., Teng J.G. (2007) Near-surface mounted FRP reinforcement: An emerging technique for strengthening structures. Composites Part B: Engineering (38) p119-143

[22] Westergaard H. M. and Slater W. A. (1921) Moments and stresses in slabs. J. Am. Conc. Inst. Proc.,1921, 17, pp415-538.

[23] Ockleston A. J. (1955) Load tests on a three-storey reinforced concrete building in Johannesburg. The Structural Engineer, 1955, 33, Oct. 304-322.

[24] Ockleston A. J. (1958) Arching action in reinforced concrete slabs. The Structural Engineer, 1958, 36, No. 6, 197-201.

[25] McDowell, E.L., McKee, K.E., Sevin, E. (1956) Arching Action Theory of Masonry Walls. Proceedings of the American Society of Civil Engineers, Vol. 82, No. ST2, pp915-1 - 918-18.

[26] Park R. (1964a) Ultimate strength of rectangular concrete slabs under shortterm uniform loading with edges restrained against lateral movement. Proceedings of the Institution of Civil Engineers, Volume 28, June, pp125-150.

[27] Park, R, (1964b) Tensile membrane behaviour of uniformly loaded rectangular reinforced concrete slabs with fully restrained edges. Magazine of Concrete Research, Vol. 16, Issue 46, March 1964, pp39-44.

[28] Park, R., (1964c) The ultimate strength and long-term behaviour of uniformly loaded, two-way concrete slabs with partial lateral restraint at all edges. Magazine of Concrete Research, Vol. 16, Issue 48, September 1964, pp139-152.

[29] Rankin G.I.B. (1982) Punching failure and compressive membrane action in reinforced concrete slabs. PhD Thesis, Queen's University Belfast.

[30] Rankin G.I.B., Long A.E. (1997) Arching action strength enhancement in laterally-restrained slab strips, Proceedings of the Institution of Civil Engineers: Structures and Buildings, Vol. 122, Issue 4 p461-467.

[31] Taylor, S. E. (2000) Compressive Membrane Action in High Strength Concrete Bridge Deck Slabs. PhD Thesis, Queen's University Belfast. 
[32] Taylor S.E., Rankin G.I.B., Cleland D.J. (2001) Arching Action in High Strength Concrete Slabs. Proceedings of the Institution of Civil Engineers: Structures and Buildings Vol. 146, Issue 4, p353-362.

[33] Taylor, S.E., Rankin G.I.B. and Cleland D.J. (2002) Guide to compressive membrane action in concrete bridge decks. 1st Ed. Camberley, UK: Concrete Bridge Development Group.

[34] Taylor S.E., Mullin B. (2005) Arching Action in FRP Reinforced Concrete Slabs. Construction and Building Materials (20) p71-80.

[35] Zheng Y., Robinson D., Taylor S., Cleland D., Shaat A. (2008) Analysis of Compressive Membrane Action in Concrete Slabs, Proceedings of the Institution of Civil Engineers: Bridge Engineering, Vol. 161, Issue 1, p 21-31, March 2008.

[36] Tharmarajah, G. (2011) Compressive Membrane Action in Fibre Reinforced Polymer (FRP) Reinforced Concrete Slabs. PhD Thesis, Queen's University Belfast.

[37] Asplund, S. O., (1949) Strengthening Bridge Slabs with Grouted Reinforcement. Journal of the American Concrete Institute, V20 No. 5, Jan pp. 397406

[38] Rasheed H.A., Harrison R.R., Peterman R.J., Alkhrdaji T. (2010) Ductile strengthening using externally bonded and near surface mounted composite systems. Composite Structures (92) p2379-2390.

[39] Micelli, F. and De Lorenzis, L., (2012) Near-surface mounted flexural strengthening of reinforced concrete beams with low concrete strength. Proceedings of the Institution of Civil Engineers: Construction Materials, Volume 166, Issue CM5, pp295 - 303.

[40] O'Connor, J Alamplali, S Aref A and Trianafilou L (2011) Strategic Development and Deployment of a Composite Bridge Deck, Proceedings of the International conference on Advanced Composites in Construction, UK, 2011.

[41] Militky, J.K., Vladimir, (1996) Ultimate Mechanical Properties of Basalt Filaments. Textile Research Journal, 66(4): p.225-229 
[42] American Chemical Society (2003) High Performance Carbon Fibers. [ONLINE]

Available

at:

http://www.acs.org/content/acs/en/education/whatischemistry/landmarks/carbonfiber s.html. [Accessed 17 January 14].

[43] Bacon, R. (1960) Growth, Structure and Properties of Graphite Whiskers, Journal of Applied Physics, Vol. 31, No. 2, Feb 1960, p 283-290

[44] BD 81/02, (2007) Use of compressive membrane action in bridge decks, Design Manual for Roads and Bridges, Vol. 3, Section 4, Part 20. London: United Kingdom Department for Transport, Highways Agency

[45] American Association of State Highway and Transportation Officials (AASHTO) (2012) Load and Resistance Factor Design (LRFD) Bridge Design Specifications. Washington, D.C, USA

[46] CAN/CSA-S6-06 (2006) Canadian Highway Bridge Design Code, Canadian Standards Association, Toronto, Ontario, Canada, November 2006

[47] Zheng Y. (2008) Modelling of Compressive Membrane Action in Bridge Decks. PhD Thesis, Queen's University Belfast.

[48] BS EN 12350-2 (2009) Testing fresh concrete. Slump-test, London: BSI Group

[49] BS EN 12390-1 (2012) Testing hardened concrete. Shape, dimensions and other requirements for specimens and moulds, London: BSI Group

[50] BS EN 12390-3 (2009) Testing hardened concrete. Compressive strength of test specimens, London: BSI Group

[51] BS EN 12390-6 (2009) Testing hardened concrete. Tensile splitting strength of test specimens, London: BSI Group

[52] BS EN 12390-4 (2000) Testing hardened concrete. Compressive strength. Specification for testing machines, London: BSI Group

[53] BS 4449 (2005) Steel for the reinforcement of concrete - Weldable reinforcing steel - Bar, coil and decoiled product - Specification, London: BSI Group

[54] BS EN ISO 15630-1 (2010) Steel for the reinforcement and prestressing of concrete. Test methods. Reinforcing bars, wire rod and wire, London: BSI Group 
[55] Hognestad, E. (1952) Fundamental concepts in ultimate load design of reinforced concrete members, ACI Proceedings, Vol.48, No.10, June 1952, pp.809832.

[56] Thorenfeldt, E., Tomaszewicz, A. and Jensen, J.J. (1987) Mechanical Properties of High Strength Concrete and Application in Design. Proceedings of the Symposium on Utilization of High Strength Concrete, Tapir, Trondheim, Norway, pp 149-159.

[57] Feenstra, P.H., Rots, J. G., A Arnesen, Teigen, J. G., Hoiseth, K., V. (1998) A $3 \mathrm{D}$ constitutive model for concrete based on a co-rotational concept. In The EURO-C 1998 Conference On Computational Modelling Of Concrete Structures. Badgastein, Austria, 31 March - 3 April 1998. Netherlands: A. A. Balkema Rotterdam Brookfield. pp13-22.

[58] Report of a Concrete Society Working Party (2004) Influence of tension stiffening on deflection of reinforced concrete structures, Technical Report No. 59, UK, 2004, pp35.

[59] MacGinley, T. J. and B. S. Choo (1994) Reinforced Concrete Design Theory and Examples. 2nd Ed. United Kingdom: E \& FN Spon. pp112

[60] ACl Committee 318-11 (2011) Building Code Requirements for Structural Concrete (ACI 318-11) and Commentary (ACI 318R-11), American Concrete Institute, Farmington Hills, Michigan, USA.

[61] ACl 440.2R-17 (2017) Guide for the Design and Construction of Externally Bonded FRP Systems for Strengthening Concrete Structures, American Concrete Institute, Michigan, USA

[62] BS EN 1990 (2002) Eurocode 2. Eurocode - Basis of structural design, London: BSI Group

[63] BS EN 1992-1-1 (2004) Eurocode 2. Design of concrete structures. General rules and rules for buildings, London: BSI Group 